\title{
Perturbation Theory for Almost-Periodic Potentials I: One-Dimensional Case
}

\author{
Leonid Parnovski $^{1}$ (D), Roman Shterenberg ${ }^{2}$ \\ 1 Department of Mathematics, University College London, Gower Street, London WC1E 6BT, UK. \\ E-mail: Leonid@math.ucl.ac.uk \\ 2 Department of Mathematics, University of Alabama at Birmingham, 1300 University Blvd., Birmingham, \\ AL 35294, USA. E-mail: shterenb@math.uab.edu
}

\begin{abstract}
We consider the family of operators $H^{(\varepsilon)}:=-\frac{d^{2}}{d x^{2}}+\varepsilon V$ in $\mathbb{R}$ with almostperiodic potential $V$. We study the behaviour of the integrated density of states (IDS) $N\left(H^{(\varepsilon)} ; \lambda\right)$ when $\varepsilon \rightarrow 0$ and $\lambda$ is a fixed energy. When $V$ is quasi-periodic (i.e. is a finite sum of complex exponentials), we prove that for each $\lambda$ the IDS has a complete asymptotic expansion in powers of $\epsilon$; these powers are either integer, or in some special cases half-integer. These results are new even for periodic $V$. We also prove that when the potential is neither periodic nor quasi-periodic, there is an exceptional set $\mathcal{S}$ of energies (which we call the super-resonance set) such that for any $\sqrt{\lambda} \notin \mathcal{S}$ there is a complete power asymptotic expansion of IDS, and when $\sqrt{\lambda} \in \mathcal{S}$, then even two-terms power asymptotic expansion does not exist. We also show that the super-resonant set $\mathcal{S}$ is uncountable, but has measure zero. Finally, we prove that the length of any spectral gap of $H^{(\varepsilon)}$ has a complete asymptotic expansion in natural powers of $\varepsilon$ when $\varepsilon \rightarrow 0$.
\end{abstract}

\section{Introduction}

We consider the operator

$$
H=H^{(\varepsilon)}:=-\Delta+\varepsilon V,
$$

where $\varepsilon>0$ is a small parameter and $V$ is a real-valued almost-periodic potential. We are interested in various quantitative and qualitative spectral properties of $H$ as $\varepsilon \rightarrow 0$, and this paper is the first one in a series of articles devoted to the study of these properties of $H$ under various assumptions. In this paper, we assume that the dimension $d=1$, so that

$$
H=H^{(\varepsilon)}:=-\frac{d^{2}}{d x^{2}}+\varepsilon V
$$

The quantities we will be interested in are: the length of the spectral gaps, and the integrated density of states (IDS) $N\left(\lambda ; H^{(\varepsilon)}\right)$ when the spectral variable $\lambda$ is fixed; we are going to study the behaviour of these quantities as $\varepsilon \rightarrow 0$. 
The first problem we study is as follows. Let $\lambda \in \mathbb{R}$ be a fixed number and consider the behaviour of the IDS of $H^{(\varepsilon)}$ at $\lambda$ when $\varepsilon \rightarrow 0$. Questions of this nature (how the value of IDS at a fixed energy depends on the value of a small coupling constant) have arisen in our study of perturbations of Landau Hamiltonians by almost-periodic potentials. Despite the slightly esoteric feel of this type of questions, we believe they are more natural than it may seem at the first sight, especially given that the answers are quite surprising. Let us briefly describe the effects happening in dimension one; we are going to devote the second paper in this series to discuss the multidimensional case, where the results are even more unexpected. Suppose first that $V$ is quasi-periodic (i.e. $V$ is a finite linear combination of trigonometric functions). Then, whenever $\lambda$ is not a square of a frequency, there is a complete asymptotic expansion of $N\left(\lambda ; H^{(\varepsilon)}\right)$ in integer powers of $\varepsilon$. Suppose, $\lambda=\theta^{2} \neq 0$, where $\theta$ is a frequency. Then the type of the expansion will depend on the relationship between $\tau$ (the constant Fourier coefficient of $V$ ) and $v$ (the Fourier coefficient at $e^{i 2 \theta x}$ ). First we notice that, as we will show in this paper, there is a spectral gap of $H^{(\varepsilon)}$ around $\theta^{2}$ of length $\sim 2 \nu \varepsilon$. Therefore, if $|\tau|<|\nu|$, then the point $\lambda+\tau \varepsilon$ stays inside this gap and, as a result, the IDS does not depend on $\varepsilon$ when $\varepsilon$ is small. If, on the other hand, $|\tau|>|\nu|$, then the shift by $\tau \varepsilon$ pushes our point $\lambda$ well outside the spectral gap, and we obtain the standard asymptotic expansion in integer powers of $\varepsilon$. The most interesting case is $|\tau|=|\nu|$, when the point $\lambda+\tau \varepsilon$ is approximately at the edge of the spectral gap. In this case generically the answer will depend on the sign of $\tau$. For one value of this sign the point $\lambda+\tau \varepsilon$ is still located in the gap and so the IDS is constant. However, for the opposite value of the sign of $\tau$ the point $\lambda+\tau \varepsilon$ will be pushed just outside the gap and, as a result, the IDS will have a complete expansion in half-integer powers of $\varepsilon$ (where we define half-integers as $(\mathbb{Z} / 2) \backslash \mathbb{Z}$ ). Similar situation happens when we look at the point $\lambda=0$ : we have expansion in half-integers whenever $\tau<0$; otherwise, the expansion is in integers. The bottom line is, if $V$ is quasi-periodic, then for all $\lambda$ we have a complete asymptotic expansion of $N\left(\lambda ; H^{(\varepsilon)}\right)$ as $\varepsilon \rightarrow 0$, which contains either integer, or half-integer powers of $\varepsilon$.

An interesting phenomenon occurs when we look at this problem in the 'proper' almost-periodic setting, for example, when all the Fourier coefficients are non-zero. In this case there is a substantial set $\mathcal{S}$ such that for $\lambda^{1 / 2} \in \mathcal{S}$ there is no asymptotic expansion of $N\left(\lambda ; H^{(\varepsilon)}\right)$ at all; in fact, there are uncountably many values of $\lambda$ for which the remainder $N\left(\lambda ; H^{(\varepsilon)}\right)-N\left(\lambda ; H^{(0)}\right)$ is not even asymptotically equivalent to any power of $\varepsilon$. This set (which we call the super-resonance set) is therefore uncountable, but has measure zero; the interesting feature of this set is that it is present no matter how quickly the Fourier coefficients of $V$ go to zero-whether $V$ is smooth, or analytic, the super-resonant set without the asymptotic expansion of IDS is always uncountable (but perhaps its dimension may depend on the smoothness of $V$ ).

The second problem we consider is as follows. It has been noticed by Arnold, [1] that if $H$ is a Hill operator (1.2) with $V$ being a finite trigonometric periodic polynomial

$$
V=\sum_{j=-n}^{n} a_{j} e^{i 2 j x},
$$

then the size of the spectral gap around the point $m^{2}, m \in \mathbb{N}$ is at most $C_{m, n} \varepsilon^{-[-m / n]}$, where $[s]$ is the largest integer not bigger than $s$. If the sum in (1.3) is infinite, then the size of any gap is (generically) proportional to $\varepsilon$. It turns out that in the periodic case it is not very difficult to prove more: that the size of each spectral gap enjoys a complete asymptotic expansion in natural powers of $\varepsilon$, see e.g. [2]. Our second theorem is the 
extension of these results to the case of almost-periodic potentials: we prove that the length of each spectral gap has a complete asymptotic expansion in natural powers of $\varepsilon$. We also prove similar expansions for the upper and lower ends of each spectral gap. The leading power in each expansion will depend on whether the potential is quasi-periodic or almost-periodic. In the quasi-periodic case the leading power of the length of the gap opened around the square of each frequency $\theta$ will increase together with the order of $\theta$ (see the next section for the precise definitions and formulation of the results), whereas in the almost-periodic setting when no Fourier coefficients vanish, all expansions begin with the first power of $\varepsilon$. These expansions are formally uniform, but effectively they are not, because the higher the order of a frequency $\theta$ is, the smaller $\varepsilon$ we need to choose to 'see' the expansion of the length of the gap generated by $\theta$ (i.e. if we choose $\varepsilon$ not very small, then the remainder in the expansion will be larger than the asymptotic terms).

Somewhat similar problems were considered in [3] and, in the discrete setting, in [4] (see also [5] and references there). However, there is a significant difference between these papers and our results. In these papers the authors have fixed $\varepsilon$ and studied the behaviour of the gap length as a function of the 'natural label' of the gap (corresponding, roughly, to what we call the order of a frequency, see below for details). So, they were able to obtain information about all gaps simultaneously, but this information was either bounds (upper and lower), or one asymptotic term, whereas we obtain more detailed information (complete asymptotic expansion) about smaller number of gaps.

The method we use for obtaining our results is a version of the gauge transform method used in $[7,8]$. The only difference is that in $[7,8]$ we had fixed coupling constant and assumed that the energy $\lambda$ was large (so that the small parameter was $\lambda^{-1}$ ), whereas in the present paper the small parameter is the coupling constant $\varepsilon$. This difference is not essential, so the construction of the gauge transform can be performed almost word-toword as it is done in [7,8]. This method allows us to find two operators, $H_{1}$ and $H_{2}$ so that $H_{1}$ is unitarily equivalent to $H, H_{2}$ is close to $H_{1}$ in norm, and $H_{2}$ is almost diagonal (in the sense that most of the off-diagonal matrix coefficients of $H_{2}$ vanish). For the sake of completeness, we have written the details of the gauge transform construction relevant to our setting in "Appendix"; in the main body of the paper we will give a brief description of the method and use the relevant properties of $H_{1}$ and $H_{2}$ without proof.

The structure of the rest of the paper is as follows: in the next section we will give all the necessary definitions and formulate the main results. In Sect. 3 we will discuss the quasi-periodic operators, and in Sect. 4 the almost-periodic operators. Finally, in "Appendix" we will describe the method of the gauge transform.

\section{Notation and Main Results}

We will consider two types of the potential $V$. The first type is quasi-periodic potentials:

$$
V=\sum_{\theta \in \Theta} \hat{V}_{\theta} \mathbf{e}_{2 \theta} .
$$

Here,

$$
\mathbf{e}_{\theta}(x):=e^{i \theta x}
$$

$\hat{V}_{\theta}$ are complex numbers (called the Fourier coefficients of $V$; since $V$ is real, we have

$\hat{V}_{-\theta}=\overline{\hat{V}}_{\theta}$ ), and $\Theta=\Theta(V) \subset \mathbb{R}^{d}$ is a finite set, called the set of frequencies (or rather half-frequencies; the factor 2 is used purely for convenience) of $V$. We assume without loss of generality that $\Theta$ is symmetric about the origin and contains it. Denote 
by $l$ the number of independent elements in $\Theta$ (so that $|\Theta|=2 l+1$ ). For each natural $L$ we denote $\Theta_{L}:=\Theta+\Theta+\cdots+\Theta$ (the algebraic sum of $L$ copies of $\Theta$ ) and put $\Theta_{\infty}:=\cup_{L} \Theta_{L}$. When $\theta \in \Theta_{\infty}$, we denote by $Z(\theta)$ the smallest number $L$ for which $\theta \in \Theta_{L}$ and call this number the order of the frequency $\theta$. We put

$$
T_{L}:=\Theta_{L} \backslash \Theta_{L-1} .
$$

A simple combinatorial estimate shows that

$$
\# \Theta_{L} \leq(3 L)^{3 l} \text {. }
$$

We also put $\tau:=\hat{V}_{0}, \Theta^{\prime}:=\Theta \backslash\{0\}$ and $V^{\prime}:=V-\tau$, so that

$$
V^{\prime}=\sum_{\theta \in \Theta^{\prime}} \hat{V}_{\theta} \mathbf{e}_{2 \theta}
$$

The second type of potentials we are going to consider are smooth almost-periodic, by which we mean that $\Theta$ is still a finite set, but we have

$$
V=\sum_{\theta \in \Theta_{\infty}} \hat{V}_{\theta} \mathbf{e}_{2 \theta}
$$

with

$$
\left|\hat{V}_{\theta}\right| \ll m^{-P}
$$

for $\theta \in T_{m}$ and arbitrary positive $P$.

We also assume that $\Theta$ satisfies the diophantine condition, i.e. for $\theta \in \Theta_{m}$ we have $|\theta| \gg m^{-P_{0}}$, where $P_{0}>0$ is fixed.

In either of these two cases (quasi- or almost-periodic potentials) we also assume (as we can do without loss of generality) that

$$
\|V\|_{2}:=\left(\sum_{\theta \in \Theta_{\infty}}\left|\hat{V}_{\theta}\right|^{2}\right)^{1 / 2}<1 / 100 .
$$

Our first main result concerns the spectral gaps.

Theorem 2.1. Suppose, $V$ is either quasi-periodic, or infinitely smooth almost-periodic and satisfies all the above assumptions. Suppose, $\theta \in \Theta_{\infty}^{\prime}$. Then for sufficiently small $\varepsilon$ operator $H$ has a (possibly trivial) spectral gap around $|\theta|^{2}$, the length of which, as well as its upper and lower ends, have complete asymptotic expansions in natural powers of $\varepsilon$. If $\hat{V}_{\theta} \neq 0$ then the asymptotic expansion for the upper (lower) end of the gap starts with $|\theta|^{2} \pm\left|\hat{V}_{\theta}\right| \varepsilon+O\left(\varepsilon^{2}\right)$.

Remark 2.2. If $\hat{V}_{\theta}=0$, we cannot guarantee that an expansion for the gap-length is always non-trivial, i.e. it could happen, in principle, that the length of the gap is $O\left(\varepsilon^{+\infty}\right)$.

The next result involves two quantities, $s_{2}(0)$ and $g_{2}(0)$ which will be defined in the next section (in formula (3.24)). Throughout the paper we use the convention that each time we use letters $a_{j}$ (or $a_{j}(\lambda)$ ) for coefficients in asymptotic expansions, the exact values of these coefficients could be different. The same refers to the use of $C$ which can mean a different positive constant each time we use it. 
Theorem 2.3. Suppose, $V$ is quasi-periodic. Then for sufficiently small $\varepsilon>0$ (i.e. $\varepsilon<\varepsilon_{0}$, where $\varepsilon_{0}>0$ depends on $V$ and $\lambda$ ) the following holds:

(i) For $\lambda<0$ we have $N(\lambda ; H)=0$.

(ii) For $\lambda>0$ and $\sqrt{\lambda} \notin \Theta_{\infty}$ we have

$$
N(\lambda ; H) \sim \pi^{-1} \sqrt{\lambda}+\sum_{p=1}^{\infty} a_{p}(\lambda) \varepsilon^{p} .
$$

(iii) For $\lambda=\left|\theta_{0}\right|^{2}$ with $\theta_{0} \in \Theta^{\prime}$ (i.e. $v:=\hat{V}_{\theta_{0}} \neq 0$ ) there are the following options:

(a) If either $|\tau|<|\nu|$, or $|\tau|=|\nu|$ and $\left[s_{2}(0) \tau-\mathfrak{R}\left(\nu \bar{g}_{2}(0)\right)\right]<0$, then

$$
N(\lambda ; H)=\pi^{-1}\left|\theta_{0}\right| ;
$$

(b) If $|\tau|>|\nu|$, then

$$
N(\lambda ; H) \sim \pi^{-1}\left|\theta_{0}\right|+\sum_{p=1}^{\infty} a_{p}(\lambda) \varepsilon^{p}, \quad a_{1}(\lambda)<0 ;
$$

(c) If $|\tau|=|\nu|$ and $\left[s_{2}(0) \tau-\Re\left(\nu \bar{g}_{2}(0)\right)\right]>0$, then

$$
N(\lambda ; H) \sim \pi^{-1}\left|\theta_{0}\right|+\varepsilon^{3 / 2} \sum_{p=0}^{\infty} a_{p}(\lambda) \varepsilon^{p}, \quad a_{0}(\lambda)<0
$$

(d) If $|\tau|=|\nu|$ and $\left[s_{2}(0) \tau-\Re\left(\nu \bar{g}_{2}(0)\right)\right]=0$, then

$$
N(\lambda ; H) \sim \pi^{-1}\left|\theta_{0}\right|+\varepsilon^{k / 2} \sum_{p=0}^{\infty} a_{p}(\lambda) \varepsilon^{p}, \quad a_{0}(\lambda)<0,
$$

with some natural $k \geq 4$ including, possibly, $k=\infty$ (the latter means that $N(\lambda ; H)=$ $\left.\pi^{-1}\left|\theta_{0}\right|+o\left(\varepsilon^{+\infty}\right)\right)$.

(iv) For $\lambda=\left|\theta_{0}\right|^{2}$ with $\theta_{0} \in \Theta_{\infty} \backslash \Theta$ (i.e. $v:=\hat{V}_{\theta_{0}}=0$ ) there are the following options:

(a) If $\tau=0$, and $s_{2}^{2}(0)<\left|g_{2}(0)\right|^{2}$, then

$$
N(\lambda ; H)=\pi^{-1}\left|\theta_{0}\right|
$$

(b) If $|\tau|>0$, then

$$
N(\lambda ; H) \sim \pi^{-1}\left|\theta_{0}\right|+\sum_{p=1}^{\infty} a_{p}(\lambda) \varepsilon^{p}, \quad a_{1}(\lambda)<0 .
$$

(c) If $\tau=0$ and $s_{2}^{2}(0)>\left|g_{2}(0)\right|^{2}$, then

$$
N(\lambda ; H) \sim \pi^{-1}\left|\theta_{0}\right|+\sum_{p=2}^{\infty} a_{p}(\lambda) \varepsilon^{p}, \quad a_{2}(\lambda)<0
$$


(d) If $\tau=0$ and $s_{2}^{2}(0)=\left|g_{2}(0)\right|^{2}$, then

$$
N(\lambda ; H) \sim \pi^{-1}\left|\theta_{0}\right|+\varepsilon^{k / 2} \sum_{p=0}^{\infty} a_{p}(\lambda) \varepsilon^{p}, \quad a_{0}(\lambda)<0,
$$

with some natural $k \geq 5$ including, possibly, $k=\infty$.

(v) Suppose, $\lambda=0$. Then there are the following options:

(a) If $\tau>0$, then $N(0 ; H)=0$;

(b) If $\tau<0$, then

$$
N(0 ; H) \sim \varepsilon^{1 / 2}\left(\pi^{-1}|\tau|^{1 / 2}+\sum_{p=1}^{\infty} a_{p} \varepsilon^{p}\right), \quad a_{1}>0
$$

(c) If $\tau=0$, then

$$
N(0 ; H) \sim \varepsilon \sum_{p=0}^{\infty} a_{p} \varepsilon^{p}, \quad a_{0}>0 .
$$

Finally, the following result holds for almost-periodic potentials.

Theorem 2.4. Suppose, $V$ is infinitely smooth almost-periodic, but not periodic, and $\hat{V}_{\theta} \neq 0$ for any $\theta \in \Theta_{\infty}^{\prime}$. Then there exists a set $\mathcal{S}$ (which we call a super-resonance set) such that a complete power asymptotic expansion of $N(\lambda ; H)$ exists if and only if $\lambda \notin \mathcal{S}$. The set $\mathcal{S}$ is uncountable and has measure zero.

Remark 2.5. As we will see in the proof, there are uncountably many values of $\lambda$ for which the difference $N\left(\lambda ; H^{(\varepsilon)}\right)-N\left(\lambda ; H^{(0)}\right)$ properly oscillates between $C_{1} \varepsilon^{j}$ and $C_{2} \varepsilon^{j}$, where $C_{1} \neq C_{2}$ and $j$ equals 1 or 2 .

We will think of a point $\xi \in \mathbb{R}$ as the exponential function $\mathbf{e}_{\xi}(x):=e^{i \xi x}$ lying in the Besikovich space $B_{2}(\mathbb{R})$ (the collection of all formal countable linear combinations of $\left\{\mathbf{e}_{\xi}\right\}$ with square-summable coefficients). Then for arbitrary pseudo-differential operator $W$ with symbol (in a left quantisation) $w=w(\xi, x)$ being quasi-periodic in $x$,

$$
w(\xi, x)=\sum_{\theta \in \Theta} \mathbf{e}_{2 \theta}(x) \hat{w}(\xi, \theta),
$$

we have

$$
W \mathbf{e}_{\xi}=\mathbf{e}_{\xi} w(\xi, x) \text {. }
$$

Thus, we can think of the Fourier coefficients $\hat{w}(\theta, \xi)$ of the symbol as the matrix element of $W$ joining $\xi$ and $\xi+2 \theta$ :

$$
\hat{w}(\xi, \theta)=\left\langle W \mathbf{e}_{\xi}, \mathbf{e}_{\xi+2 \theta}\right\rangle_{B_{2}(\mathbb{R})} .
$$

In our paper [7] it is explained that instead of working with operators acting in $L_{2}(\mathbb{R})$, we can consider operators with the same symbol acting in $B_{2}(\mathbb{R})$ and work with them. This will not change the spectral properties we are studying in our paper (for example, the spectrum as a set is the same whether our operator acts in $L_{2}(\mathbb{R})$ or $B_{2}(\mathbb{R})$ ). 


\section{Quasi-Periodic Potential}

In this section we assume that the potential $V$ is quasi-periodic, i.e. that (2.1) holds.

3.1. Gauge transform: general description. First of all, we give a brief outline of the construction of the gauge transform of our operator. The details of this construction are similar to those in [7]; for the sake of completeness, we present them in "Appendix". Let us fix a natural number $N$. All the constructions are going to depend on the choice of $N$, but we will often omit writing $N$ as the variable. Applying the gauge transform leads to a pair of operators, $H_{1}=H_{1}^{(\varepsilon)}$ and $H_{2}=H_{2}^{(\varepsilon)}$ so that $H_{1}=U H U^{-1}$ is unitarily equivalent to $H ; H_{1}$ and $H_{2}$ are close in norm, more precisely,

$$
|| H_{1}-H_{2} \| \ll \varepsilon^{N}
$$

and $\mathrm{H}_{2}$ is almost diagonal in the sense that it can be decomposed into a direct integral with all fibres being finite dimensional (moreover, as we will see, the dimension of all fibres will be 1 or 2). Also, the frequencies of $H_{2}$ are inside the set $\Theta_{3 N}$. Here, the coefficient 3 technically appears in the gauge transform approach (see "Appendix"). It reflects the fact that one has to make slightly more than $N$ steps to achieve the error of order $\varepsilon^{N}$. Once we have constructed these operators, it turns out that we can study spectral characteristics of $\mathrm{H}$ by means of studying the corresponding spectral characteristics of $\mathrm{H}_{2}$. Indeed, the spectra of $H$ and $H_{1}$ are the same, and so are the lengths of the spectral gaps. Also, the lengths of the spectral gaps of $H_{1}$ and $H_{2}$ differ by at most $\varepsilon^{N}$.

Concerning the IDS, it was proved in [7] that

$$
N\left(\lambda ; H_{1}\right)=N(\lambda ; H)
$$

and

$$
\left|N\left(\lambda ; H_{2}\right)-N\left(\lambda ; H_{1}\right)\right| \ll \varepsilon^{(N+1) / 2} .
$$

More precisely, we have shown in [7] that the immediate consequence of (3.1) is

$$
N\left(\lambda-\varepsilon^{N} ; H_{2}\right) \leq N\left(\lambda ; H_{1}\right) \leq N\left(\lambda+\varepsilon^{N} ; H_{2}\right),
$$

and now (3.3) would follow once we establish expansion (4.6) for $N\left(\lambda ; H_{2}^{(\varepsilon)}\right)$.

We also define

$$
H^{\prime}:=H_{0}+V^{\prime}=H-\varepsilon \tau, \quad H_{j}^{\prime}:=H_{j}-\varepsilon \tau, \quad j=1,2,
$$

and notice the obvious property

$$
N\left(\lambda ; H_{j}\right)=N\left(\lambda-\mu ; H_{j}-\mu\right),
$$

so if we put

$$
\mu:=\varepsilon \tau,
$$

we have

$$
N\left(\lambda ; H_{j}\right)=N\left(\lambda-\mu ; H_{j}^{\prime}\right) .
$$

This trivial consideration is important for understanding of some of the effects described later. 
Now we choose a small positive number $\delta=\delta(N)$, to be specified later and for each non-zero frequency $\theta \in \Theta^{\prime}\left(H_{2}\right)$ we put

$$
R(\theta)=R(\theta ; \delta):=\left\{\xi \in \mathbb{R},\left|(\xi+2 \theta)^{2}-\xi^{2}\right|<\delta\right\}=\left(-\theta-\frac{\delta}{4|\theta|},-\theta+\frac{\delta}{4|\theta|}\right) .
$$

Next, let $\psi=\psi(\xi)$ be a standard smooth non-negative cut-off function satisfying $\operatorname{supp} \psi \subset[-1 / 2,1 / 2]$ and $\psi(\xi)=1$ for $\xi \in[-1 / 4,1 / 4]$, and let $\varphi:=1-\psi$. We put

$$
\varphi_{\theta}(\xi):=\varphi\left((\xi+\theta) 4|\theta| \delta^{-1}\right) \text {. }
$$

Note that

$$
R(-\theta)=-R(\theta), \quad \varphi_{-\theta}(-\xi)=\varphi_{\theta}(\xi)
$$

We also put

$$
\tilde{\chi}_{\theta}(\xi):=\varphi_{\theta}(\xi)\left(|\xi+2 \theta|^{2}-|\xi|^{2}\right)^{-1}=\frac{\varphi_{\theta}(\xi)}{4(\xi+\theta) \theta}
$$

when $\theta \neq 0$, and $\tilde{\chi}_{0}(\xi)=0$.

The region $R(\theta)$ is called the resonance zone corresponding to $\theta$. Since (for fixed $N$ ) the number of resonance zones is finite and the length of them goes to zero, it implies that for sufficiently small $\delta$ these zones do not intersect. We also denote by

$$
\mathbf{R}(\delta):=\cup_{\theta \in \Theta^{\prime}\left(H_{2}\right)} R(\theta ; \delta)
$$

the 'overall' resonant set corresponding to $\varepsilon$; we obviously have

$$
\mathbf{R}\left(\delta_{1}\right) \subset \mathbf{R}\left(\delta_{2}\right)
$$

for $\delta_{2}>\delta_{1}$.

In what follows we always assume that $\delta(N)$ is sufficiently small so that different resonance zones $R(\theta ; \delta)$ do not intersect for all $\theta \in \Theta_{9 N}^{\prime}$; we also take $\varepsilon$ so small that $\varepsilon \leq \delta^{2}$.

Remark 3.1. It is not difficult to see that in case when $\Theta^{\prime}$ satisfies Diophantine condition on frequencies, the parameter $\delta(N)$ can be chosen to be $c^{N}$ with some constant $c=c(\Theta)$ with all constructions and statements of Sect. 3 being valid.

The important property of the operator $\mathrm{H}_{2}$ established in "Appendix" is as follows: the Fourier coefficients $\hat{h}_{2}(\xi ; \theta)$ satisfy

$$
\hat{h}_{2}(\xi ; \theta)=0, \text { if } \theta \neq 0 \text { and } \xi \notin R(\theta) .
$$

This property implies that if a point $\xi$ lies outside all the resonance zones, then the onedimensional subspace spanned by the corresponding $\mathbf{e}_{\xi}$ is invariant with respect to $\mathrm{H}_{2}$. If, on the other hand, for some (unique) $\theta$ we have $\xi \in R(\theta)$, then the two-dimensional subspace spanned by $\mathbf{e}_{\xi}$ and $\mathbf{e}_{\xi+2 \theta}$ is invariant with respect to $H_{2}$.

We have the following further properties of the symbol of $\mathrm{H}_{2}$. The non-vanishing Fourier coefficients $\hat{h}_{2}(\xi ; \theta)$ must have $\theta \in \Theta_{3 N}$. Each Fourier coefficient $\hat{h}_{2}(\xi ; \theta)$ is smooth outside of the end-points of $R(\theta)$. Also, we have $\hat{h}_{2}(-\xi ;-\theta)=\hat{h}_{2}(\xi ; \theta)$ and $\hat{h}_{2}(\xi ; \theta)=\overline{\hat{h}_{2}(\xi+2 \theta ;-\theta)}$. The Fourier coefficient $\hat{h}_{2}(\xi ; 0)$ satisfies

$$
\hat{h}_{2}(\xi ; 0)=|\xi|^{2}+\varepsilon \tau+f(\xi ; 0),
$$


where $f(\xi ; 0)$ is the perturbation theory correction:

$$
f(\xi ; 0)=\sum_{p=2}^{N} \varepsilon^{p} f_{p}(\xi ; 0) .
$$

Here, each $f_{p}$ is a sum of terms of the following type: each term is a product of $p$ Fourier coefficients of $V$ and $p-1$ functions $\tilde{\chi}_{\theta_{j}}$ (for details see Lemma 5.4). In particular,

$$
f_{2}(\xi ; 0)=-\sum_{\theta \in \Theta^{\prime}}\left|\hat{V}_{\theta}\right|^{2} \tilde{\chi}_{\theta}(\xi)
$$

and, assuming $\xi$ is non-resonant for all $\theta$, we have

$$
f_{2}(\xi ; 0)=-\sum_{\theta \in \Theta^{\prime}} \frac{\left|\hat{V}_{\theta}\right|^{2}}{|\xi+2 \theta|^{2}-|\xi|^{2}} .
$$

Note that (3.15) implies that for $\xi>0$ and sufficiently small (depending on $\xi$ ) $\varepsilon$ we have

$$
\frac{\partial}{\partial \xi} \hat{h}_{2}(\xi ; 0)>0 \text {. }
$$

Similarly, if $\xi \in R(\theta)$ (and $\theta \neq 0$ ), we have

$$
\hat{h}_{2}(\xi ; \theta)=\varepsilon \hat{V}_{\theta}\left(1-\varphi_{\theta}(\xi)\right)+\sum_{p=2}^{N} \varepsilon^{p} f_{p}(\xi ; \theta),
$$

where $f_{p}(\xi ; \theta)$ has a form similar to $f_{p}(\xi ; 0)$ and, in particular,

$$
f_{2}(\xi ; \theta)=-\frac{1}{2} \sum_{\theta_{1}, \theta_{2} \in \Theta^{\prime}, \theta_{1}+\theta_{2}=\theta} \hat{V}_{\theta_{1}} \hat{V}_{\theta_{2}}\left(\tilde{\chi}_{\theta_{2}}(\xi)-\tilde{\chi}_{\theta_{2}}\left(\xi+2 \theta_{1}\right)\right) .
$$

As we have stated above, there are two types of invariant subspaces of $\mathrm{H}_{2}$ :

1. If $\xi \notin \cup_{\theta \in \Theta_{3 N}} R(\theta)$, then $\mathbf{e}_{\xi}$ generates a one-dimensional invariant subspace of $\mathrm{H}_{2}$;

2. Suppose, $\xi \in R(\theta)$ for some $\theta \in \Theta_{3 N}$. Denote $\eta:=\xi+2 \theta \in R(-\theta)$. Then the two-dimensional subspace generated by $\mathbf{e}_{\xi}$ and $\mathbf{e}_{\eta}$ is invariant under $\mathrm{H}_{2}$.

3.2. Basic constructions. Let us now construct the mapping $G: \mathbb{R} \rightarrow \mathbb{R}$ defined in the following way. Suppose first that $\xi$ is non-resonant. Then we put $G(\xi):=\hat{h}_{2}(\xi ; 0)=$ $|\xi|^{2}+\varepsilon \tau+f(\xi ; 0)$; notice that for non-resonant $\xi$ we thus have $G(-\xi)=G(\xi)$. Suppose now that $\xi$ is resonant. Then it belongs to exactly one resonance zone, say $\xi \in R(-\theta)$. Consider the $2 \times 2$ matrix $M(\xi)=M_{-\theta}(\xi)$, where the diagonal elements are $\hat{h}_{2}(\xi ; 0)$ and $\hat{h}_{2}(\xi-2 \theta ; 0)$ and off-diagonal elements are $\hat{h}_{2}(\xi ;-\theta)$ and $\hat{h}_{2}(\xi-2 \theta ; \theta)$. This matrix is Hermitian and has two real eigenvalues $\lambda_{1}(M(\xi)) \leq \lambda_{2}(M(\xi))$. We define $G(\xi)=\lambda_{2}(M(\xi))$ if $\xi>0$ and $G(\xi)=\lambda_{1}(M(\xi))$ if $\xi<0$. Thus defined function $G$ is even outside the resonance zones, increasing for positive $\xi$ outside the resonance zones and is continuous outside the end-points of the resonance zones. At the end points of resonant zones it may have jumps. These jumps are caused by the fact that the pair of 


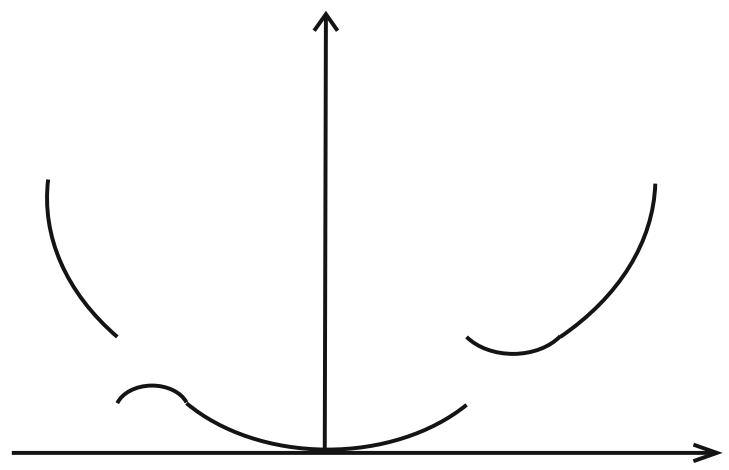

Fig. 1. The graph of $\mathrm{G}$ in the simplest case of one frequency

eigenvalues $\left(\lambda_{1}(M(\xi)), \lambda_{2}(M(\xi))\right)$ can be extended continuously outside a resonance zone, but once we have made a choice of one eigenvalue for each resonance point $\xi$, we have introduced discontinuities. A careful look at the situation convinces one that in fact if we consider two resonant zones $R( \pm \theta, \delta)$, then the function $G$ may have discontinuities only at the left endpoints, namely $\pm \theta-\frac{\delta}{4|\theta|}$, and there the jump of $G$ can be quite substantial (of order $\varepsilon$ ). At two other points $\pm \theta+\frac{\delta}{4|\theta|}$ function $G$ is continuous. See Fig. 1 for the sketch of the graph of $G$.

The most important property of $G$ is the following one: we have

$$
N\left(\lambda ; H_{2}\right)=(2 \pi)^{-1} \operatorname{meas}\{\xi, G(\xi) \leq \lambda\}=:(2 \pi)^{-1} \text { meas } \Omega_{\lambda}
$$

where we have denoted $\{\xi, G(\xi) \leq \lambda\}=: \Omega_{\lambda}=\Omega_{\lambda}(G)$. This property was proved in [7] and it immediately implies that the spectrum of $\mathrm{H}_{2}$ is

$$
\sigma\left(H_{2}\right)=\overline{\{G(\xi), \xi \in \mathbb{R}\}}
$$

Equation (3.23) shows that in order to study the spectrum of $\mathrm{H}_{2}$, we need to look at the range of $G$. Our discussions above and Fig. 1 imply the following statement:

Lemma 3.2. The range of $G$ consists of the entire semi-axis $\left[\lambda_{0},+\infty\right)$, with the possible exception of the gaps, 'generated' by the resonant zones. Each pair of zones $R( \pm \theta)$ generates at most one gap $\left(a_{-}(\theta), a_{+}(\theta)\right)$.

Later, we will obtain more precise information on the location and the length of the gaps.

Let us introduce a change of variables: we put $\xi=\theta_{0}+\zeta \in R\left(-\theta_{0}\right)$, so that $|\zeta| \leq \frac{\delta}{4\left|\theta_{0}\right|}$. We also put 


$$
\begin{aligned}
s=s(\zeta) & :=\frac{f\left(\zeta-\theta_{0} ; 0\right)+f\left(\zeta+\theta_{0} ; 0\right)}{2}=O\left(\varepsilon^{2}\right), \\
t=t(\zeta) & :=\frac{f\left(\zeta-\theta_{0} ; 0\right)-f\left(\zeta+\theta_{0} ; 0\right)}{2}=O\left(\varepsilon^{2}\right), \\
v & :=\hat{V}\left(\theta_{0}\right), \\
g=g(\zeta) & :=\hat{h}_{2}\left(\zeta+\theta_{0} ;-\theta_{0}\right)-v \varepsilon=O\left(\varepsilon^{2}\right), \\
s_{j}=s_{j}(\zeta) & :=\frac{f_{j}\left(\zeta-\theta_{0} ; 0\right)+f_{j}\left(\zeta+\theta_{0} ; 0\right)}{2} \\
t_{j}=t_{j}(\zeta) & :=\frac{f_{j}\left(\zeta-\theta_{0} ; 0\right)-f_{j}\left(\zeta+\theta_{0} ; 0\right)}{2} \\
g_{j}=g_{j}(\zeta) & :=f_{j}\left(\zeta+\theta_{0} ;-\theta_{0}\right)
\end{aligned}
$$

The diagonal elements of $M(\xi)$ are equal to $\left(\zeta-\theta_{0}\right)^{2}+(s+t)+\mu$ and $\left(\zeta+\theta_{0}\right)^{2}+$ $(s-t)+\mu, \mu:=\varepsilon \tau$ and the off-diagonal elements are $\varepsilon \nu+g$ and $\varepsilon \bar{v}+\bar{g}$.

The characteristic polynomial of the matrix $M(\xi)-\mu$ is

$$
\begin{aligned}
\chi(\sigma)= & \sigma^{2}-2 \sigma\left[\left(\zeta^{2}+\theta_{0}^{2}\right)+s\right]+\left[\left(\zeta^{2}-\theta_{0}^{2}\right)^{2}\right. \\
& \left.+2 s\left(\zeta^{2}+\theta_{0}^{2}\right)-\left|v^{2}\right| \varepsilon^{2}+4 t \zeta \theta_{0}+\left(s^{2}-t^{2}-2 \varepsilon \Re(\nu \bar{g})-|g|^{2}\right)\right]
\end{aligned}
$$

and the eigenvalues of $M(\xi)$ are

$$
\sigma_{ \pm}=\sigma_{ \pm}\left(\zeta ; \theta_{0} ; \mu\right)=\zeta^{2}+\theta_{0}^{2}+\mu+s \pm\left(\left(2 \zeta \theta_{0}-t\right)^{2}+|\varepsilon v+g|^{2}\right)^{1 / 2}
$$

Obviously, $\lambda_{2}(M(\xi))=\sigma_{+}(\zeta)$, and $\lambda_{1}(M(\xi))=\sigma_{-}(\zeta)$.

3.3. Spectral gaps. Let us find the size of the spectral gap around $\theta_{0}^{2}+\mu$. This gap is an interval $\left[\sigma_{-}^{\max }, \sigma_{+}^{\min }\right]$, where $\sigma_{-}^{\max }$ is the maximal value of $\sigma_{-}\left(\zeta ; \theta_{0} ; \mu\right)$ when $\zeta$ runs over the interval $\left[-\frac{\delta}{4\left|\theta_{0}\right|}, \frac{\delta}{4\left|\theta_{0}\right|}\right]$, and $\sigma_{+}^{\min }$ is the minimal value of $\sigma_{+}\left(\zeta ; \theta_{0} ; \mu\right)$.

Remark 3.3. It is easy to see and will be even clearer in what follows that all the objects we are interested in require detailed information only from the interior of the resonant zones. In particular, maximum value of $\sigma_{-}$and minimal value of $\sigma_{+}$are attained inside the interval $\left[-\frac{\delta}{100\left|\theta_{0}\right|}, \frac{\delta}{100\left|\theta_{0}\right|}\right]$ (assuming of course, $\varepsilon$ is small enough). This allows us to ignore cut-off functions $\varphi_{\theta}$ introduced above as they are equal to zero in the region of interest.

Recall that $|\mu| \ll \varepsilon$ and $|s|,|t|,|g| \ll \varepsilon^{2}$. Moreover, it follows from the definitions and properties of $\hat{h}_{2}$ that $t(0)=s^{\prime}(0)=0$. Thus, we can rewrite

$$
\sigma_{ \pm}=\sigma_{ \pm}\left(\zeta ; \theta_{0} ; \mu\right)=\zeta^{2}\left(1+O\left(\varepsilon^{2}\right)\right)+\theta_{0}^{2}+\mu+s(0) \pm\left(4 \zeta^{2} \theta_{0}^{2}\left(1+O\left(\varepsilon^{2}\right)\right)+|\varepsilon v+g|^{2}\right)^{1 / 2}
$$

If $\hat{h}_{2}\left(\theta_{0} ;-\theta_{0}\right)=0$, which in particular means that $v=g(0)=0$ then, obviously, $\sigma_{+}(0)=\sigma_{-}(0)=\theta_{0}^{2}+\mu+s(0)$ and thus, we have no gap. Assume now that $\hat{h}_{2}\left(\theta_{0} ;-\theta_{0}\right) \neq$ 0 . Let us use the analytic expansion for

$$
\left|\hat{h}_{2}\left(\theta_{0}+\zeta ;-\theta_{0}\right)\right|^{2}=|\varepsilon v+g|^{2}
$$

in powers of $\zeta$ in the neighbourhood of $\zeta=0$ as $(\varepsilon v+g(\zeta)) \overline{(\varepsilon v+g(\bar{\zeta}))}$. 
Remark 3.4. This complete expansion exists (both here and below) because all cut-off functions $\varphi_{\theta}$ are equal either to 0 or 1 in the regions under consideration. For more details see "Appendix", in particular, Remark 5.3 and Lemma 5.4

Thus, let us denote by $2 p, p \in \mathbb{N}$ the first non-trivial power in the expansion of $\left|\hat{h}_{2}\left(\theta_{0} ;-\theta_{0}\right)\right|^{2}$ in powers of $\varepsilon$; obviously, this power has to be even since the expression is non-negative. We put $c_{1}(\varepsilon):=\varepsilon^{-2 p}\left|\hat{h}_{2}\left(\theta_{0} ;-\theta_{0}\right)\right|^{2}, c_{1}(0)>0$. We then have

$$
|\varepsilon \nu+g|^{2}=c_{1}(\varepsilon) \varepsilon^{2 p}+c_{2}(\varepsilon) \varepsilon^{p+q} \zeta+\zeta^{2} O\left(\varepsilon^{3}\right), \quad c_{1}(0)>0, \quad q \geq p,
$$

where $c_{2}(\varepsilon)$ is analytic in $\varepsilon$ and either $q \in \mathbb{N}$ (then it is easy to see that $q \geq 2$ ), or $q=\infty$ (meaning $c_{2} \equiv 0$ ). In particular, we have $p=1$ and $c_{1}(0)=|\nu|^{2}$ for $v \neq 0$, or $p \geq 2$ for $v=0$. Suppose first that $q=+\infty$ (i.e. $c_{2} \equiv 0$ ). Then, obviously, we have $\sigma_{-}^{\max }=\sigma_{-}(0)$ and $\sigma_{+}^{\min }=\sigma_{+}(0)$, which implies that the size of the gap is exactly $2\left|\hat{h}_{2}\left(\theta_{0},-\theta_{0}\right)\right|=2 \sqrt{c_{1} \varepsilon^{2 p}}$. Thus, we can assume that $q$ is finite. Calculating derivatives, we obtain the following equations for any critical points $\zeta_{ \pm}$of $\sigma_{ \pm}$:

$$
2 \zeta\left(1+O\left(\varepsilon^{2}\right)\right)\left(4 \zeta^{2} \theta_{0}^{2}\left(1+O\left(\varepsilon^{2}\right)\right)+|\varepsilon \nu+g|^{2}\right)^{1 / 2} \pm\left(4 \zeta \theta_{0}^{2}\left(1+O\left(\varepsilon^{2}\right)\right)+c_{2} \varepsilon^{p+q}\right)=0 .
$$

We immediately see that $\zeta_{ \pm}=O\left(\varepsilon^{p+q}\right)$. Then we can rescale $\zeta=: \varepsilon^{p+q} z$ and rewrite (3.29) as follows (here we also use (3.28)):

$$
2 z\left(1+O\left(\varepsilon^{2}\right)\right) \varepsilon^{p}\left(c_{1}+O\left(\varepsilon^{2 q}\right)\right)^{1 / 2} \pm\left(4 z \theta_{0}^{2}\left(1+O\left(\varepsilon^{2}\right)\right)+c_{2}\right)=0 .
$$

This shows that the solutions $z_{ \pm}$and thus $\zeta_{ \pm}$are analytic in $\varepsilon$ with the main term $\zeta_{ \pm} \sim-\frac{c_{2} \varepsilon^{p+q}}{4 \theta_{0}^{2}}$. Plugging this into (3.26) and using the fact that $\sigma_{-}^{\max }=\sigma_{-}\left(\zeta_{-}\right)$and $\sigma_{+}^{\min }=\sigma_{+}\left(\zeta_{+}\right)$, we obtain the following lemma

Lemma 3.5. If $\hat{h}_{2}\left(\theta_{0} ;-\theta_{0}\right) \equiv 0$ (as a function of $\varepsilon$ ), then the size of the gap is $O\left(\varepsilon^{N}\right)$. If $\hat{h}_{2}\left(\theta_{0} ;-\theta_{0}\right) \not \equiv 0$, then there exists the complete asymptotic expansion for the size of the gap (up to the order $O\left(\varepsilon^{N}\right)$ ) starting with twice the first non-zero power of $\varepsilon$ in expansion (3.20) of $\left|\hat{h}_{2}\left(\theta_{0} ;-\theta_{0}\right)\right|$, i.e. either $2|v| \varepsilon$ or $2\left|f_{p}\left(\theta_{0},-\theta_{0}\right)\right| \varepsilon^{p}, p \geq 2$.

Since $N$ is arbitrary, we immediately obtain the following result (which also implies Theorem 2.1 for quasi-periodic case):

Theorem 3.6. Suppose, $V$ is quasi-periodic. If $\hat{h}_{2}\left(\theta_{0} ;-\theta_{0}\right) \equiv 0$ for each $N$, then the size of the spectral gap around $\theta_{0}^{2}$ is $O\left(\varepsilon^{\infty}\right)$. If $\hat{h}_{2}\left(\theta_{0} ;-\theta_{0}\right) \not \equiv 0$ for some $N$, then there exists the complete asymptotic expansion for the size of the gap in natural powers of $\varepsilon$; the first term of this expansion is twice the first non-zero term in expansion (3.20) of $\left|\hat{h}_{2}\left(\theta_{0} ;-\theta_{0}\right)\right|$, i.e. either $2|\nu| \varepsilon$ or $2\left|f_{p}\left(\theta_{0},-\theta_{0}\right)\right| \varepsilon^{p}, p \geq 2$.

Remark 3.7. (1) As one can see from the proof, we also have complete expansions of the upper and lower ends of each gap.

(2) We notice that while $\hat{h}_{2}$ depends on the cut-off function $\varphi, \hat{h}_{2}\left(\theta_{0},-\theta_{0}\right)$ does not. Thus, as it should be, corresponding expansion from Theorem 3.6 is independent of the particular choice of the cut-off function. 
3.4. Integrated density of states. Now let us discuss the IDS of $\mathrm{H}_{2}$. Formula (3.22) implies that in order to study the integrated density of states, we need to solve the equation

$$
G(\xi)=\lambda .
$$

In the unperturbed case (when $G(\xi)=\xi^{2}$ ) this equation has two solutions whenever $\lambda>0$. After the perturbation, this equation may have no solutions (when $\lambda$ is inside a spectral gap), or it may have one solution (when $\lambda$ is exactly at the spectral edge of $\mathrm{H}_{2}$ ). As we will see later, in other cases equation (3.31) has exactly two solutions.

If $\lambda$ is negative, the above constructions imply that $N(\lambda ; H)=0$ for sufficiently small $\varepsilon$. Suppose now that $\lambda$ is positive and $\sqrt{\lambda} \notin \Theta_{3 N}$ (in particular, $\lambda \neq 0$ ). Then, for sufficiently small $\delta$, points (both of them) $\xi$ with $\xi^{2}=\lambda$ do not belong to any resonance region; the same is true for points of the form $\lambda-\varepsilon \tau$. This, together with (3.19), implies that the equation $G(\xi)=\lambda$ has two solutions (recall that we use convention of not distinguishing two solutions that are within distance $O\left(\varepsilon^{N}\right)$ from each other), call them $G^{-1}(\lambda)>0$ and $-G^{-1}(\lambda)$. Monotonicity of $G$ implies that (again for sufficiently small $\delta$ ) the following holds: whenever $0<\eta<G^{-1}(\lambda)$, we have $G(\eta)<\lambda$, and whenever $\eta>G^{-1}(\lambda)$, we have $G(\eta)>\lambda$.

This implies that $\Omega_{\lambda}=\left[-G^{-1}(\lambda), G^{-1}(\lambda)\right]$, so that

$$
N\left(\lambda ; H_{2}\right)=\pi^{-1} G^{-1}(\lambda) .
$$

Now an easy application of the inverse function theorem and (3.16)-(3.17) imply

$$
N\left(\lambda ; H_{2}\right)=\pi^{-1} \sqrt{\lambda}+\sum_{p=1}^{N} a_{p}(\lambda) \varepsilon^{p}+O\left(\varepsilon^{N}\right) .
$$

Since $N$ is arbitrary, formulas (3.2) and (3.3) now imply

$$
N(\lambda ; H) \sim \pi^{-1} \sqrt{\lambda}+\sum_{p=1}^{\infty} a_{p}(\lambda) \varepsilon^{p} .
$$

The next case we consider is $\lambda=\theta_{0}^{2}>0$ with either $\theta_{0} \in \Theta$ (so that $\hat{V}_{\theta} \neq 0$ ), or $\theta_{0} \in \Theta_{m}$ for some $m>1$; for the sake of definiteness we will also assume that $\theta_{0}>0$. Then we have the following:

$$
\begin{aligned}
\Omega_{\lambda}= & \left\{\xi,|\xi|^{2}<\lambda, \xi \notin\left(R\left(\theta_{0}\right) \cap R\left(-\theta_{0}\right)\right)\right\} \\
& \sqcup\left\{\xi \in R\left(-\theta_{0}\right), \lambda_{2}(M(\xi)) \leq \lambda\right\} \sqcup\left\{\xi \in R\left(\theta_{0}\right), \lambda_{1}(M(\xi)) \leq \lambda\right\}
\end{aligned}
$$

(the disjoint union) and, therefore,

$$
\begin{aligned}
(2 \pi) N\left(\lambda ; H_{2}\right)= & 2 \sqrt{\lambda}-\frac{\delta}{2\left|\theta_{0}\right|}+\operatorname{meas}\left\{\xi \in R\left(-\theta_{0}\right), \lambda_{2}(M(\xi)) \leq \lambda\right\} \\
& +\operatorname{meas}\left\{\xi \in R\left(\theta_{0}\right), \lambda_{1}(M(\xi)) \leq \lambda\right\} .
\end{aligned}
$$

Note that

$$
\operatorname{meas}\left\{\xi \in R\left(\theta_{0}\right), \lambda_{1}(M(\xi)) \leq \lambda\right\}=\operatorname{meas}\left\{\xi \in R\left(-\theta_{0}\right), \lambda_{1}(M(\xi)) \leq \lambda\right\},
$$


and so

$$
\begin{aligned}
(2 \pi) N\left(\lambda ; H_{2}\right)= & 2 \sqrt{\lambda}-\frac{\delta}{2\left|\theta_{0}\right|}+\operatorname{meas}\left\{\xi \in R\left(-\theta_{0}\right), \lambda_{2}(M(\xi)) \leq \lambda\right\} \\
& +\operatorname{meas}\left\{\xi \in R\left(-\theta_{0}\right), \lambda_{1}(M(\xi)) \leq \lambda\right\} .
\end{aligned}
$$

Thus, we have to study the behaviour of the eigenvalues of $M(\xi)$ when $\xi \in R\left(-\theta_{0}\right)$.

As we have mentioned above, in order to calculate it, we need to compute the measure of the set of points $\zeta,|\zeta| \leq \frac{\delta}{4\left|\theta_{0}\right|}$ for which

$$
\zeta^{2}+(s+\mu) \pm\left(4 \zeta^{2} \theta_{0}^{2}+|v|^{2} \varepsilon^{2}-4 t \zeta \theta_{0}+t^{2}+2 \varepsilon \Re(\nu \bar{g})+|g|^{2}\right)^{1 / 2} \leq 0 .
$$

We start by assuming that $v \neq 0$, i.e. $\theta \in \Theta$.

Case 1. Assume first that $\mu \geq 0$. Then we always have

$$
\sigma_{+}-\theta_{0}^{2}=\zeta^{2}+(s+\mu)+\left(4 \zeta^{2} \theta_{0}^{2}+|\nu|^{2} \varepsilon^{2}-4 t \zeta \theta_{0}+t^{2}+2 \varepsilon \Re(\nu \bar{g})+|g|^{2}\right)^{1 / 2}>0
$$

and, thus,

$$
\operatorname{meas}\left\{\xi \in R\left(-\theta_{0}\right), \lambda_{2}(M(\xi)) \leq \lambda\right\}=0 .
$$

Therefore, we need to consider only the measure of $\zeta,|\zeta| \leq \frac{\delta}{4\left|\theta_{0}\right|}$ for which

$$
\sigma_{-}-\theta_{0}^{2}=\zeta^{2}+(s+\mu)-\left(4 \zeta^{2} \theta_{0}^{2}+|\nu|^{2} \varepsilon^{2}+2 \varepsilon \Re(\nu \bar{g})+\tilde{g}\right)^{1 / 2} \leq 0,
$$

where we have denoted

$$
\tilde{g}=\tilde{g}(\zeta):=-4 t \zeta \theta_{0}+t^{2}+|g|^{2} .
$$

Note that $\tilde{g}=O\left(\varepsilon^{4}\right)+\zeta^{2} O\left(\varepsilon^{2}\right)$. Inequality (3.41) is equivalent to

$$
\zeta^{4}+2(s+\mu) \zeta^{2}+(s+\mu)^{2} \leq 4 \zeta^{2} \theta_{0}^{2}+|\nu|^{2} \varepsilon^{2}+2 \varepsilon \Re(\nu \bar{g})+\tilde{g},
$$

or

$$
\zeta^{4}-2\left[2 \theta_{0}^{2}-(s+\mu)\right] \zeta^{2}+\left[(s+\mu)^{2}-|\nu|^{2} \varepsilon^{2}-2 \varepsilon \Re(\nu \bar{g})-\tilde{g}\right] \leq 0 .
$$

Let us formally solve (3.44) as a quadratic equation in $\zeta^{2}$. We obtain:

$$
\zeta_{ \pm}^{2}=\left[2 \theta_{0}^{2}-(s+\mu)\right] \pm \sqrt{\left[2 \theta_{0}^{2}-(s+\mu)\right]^{2}-\left[(s+\mu)^{2}-|\nu|^{2} \varepsilon^{2}-2 \varepsilon \Re(\nu \bar{g})-\tilde{g}\right]} .
$$

Expanding the square root in the RHS of (3.45), we obtain

$$
\zeta_{+}^{2}=2\left[2 \theta_{0}^{2}-(s+\mu)\right]+\sum_{j=1}^{\infty}(-1)^{j}\left(\begin{array}{c}
j \\
1 / 2
\end{array}\right)\left[2 \theta_{0}^{2}-(s+\mu)\right]^{-2 j+1}\left[(s+\mu)^{2}-|\nu|^{2} \varepsilon^{2}-2 \varepsilon \Re(\nu \bar{g})-\tilde{g}\right]^{j}
$$

and

$$
\zeta_{-}^{2}=-\sum_{j=1}^{\infty}(-1)^{j}\left(\begin{array}{c}
j \\
1 / 2
\end{array}\right)\left[2 \theta_{0}^{2}-(s+\mu)\right]^{-2 j+1}\left[(s+\mu)^{2}-|\nu|^{2} \varepsilon^{2}-2 \varepsilon \Re(\nu \bar{g})-\tilde{g}\right]^{j} .
$$

A straightforward application of the inverse function theorem implies that equation (3.46) (where the functions $s$ and $\tilde{g}$ have $\zeta_{+}$as their argument) has exactly one positive 
solution $\zeta_{+}^{+}=4 \theta_{0}^{2}+O(\varepsilon)$ and one negative solution $\zeta_{+}^{-}=-4 \theta_{0}^{2}+O(\varepsilon)$. Therefore, all points $\zeta \in\left[-\frac{\delta}{4\left|\theta_{0}\right|}, \frac{\delta}{4\left|\theta_{0}\right|}\right]$ satisfy the inequality $\zeta^{2}<\zeta_{+}^{2}$.

The situation with $\zeta_{-}$is a bit more involved. There are two further sub-cases. Suppose first that for all $\zeta \in\left[-\frac{\delta}{4\left|\theta_{0}\right|}, \frac{\delta}{4\left|\theta_{0}\right|}\right]$ we have $\left[(s+\mu)^{2}-|\nu|^{2} \varepsilon^{2}-2 \varepsilon \Re(\nu \bar{g})-\tilde{g}\right]<0$ (this happens, for example, if $\mu=\tau \varepsilon$ with $|\tau|<|\nu|$ ). Then the RHS of equation (3.47) is negative, so it has no real solutions. Thus, (3.41) holds for all $\zeta \in\left[-\frac{\delta}{4\left|\theta_{0}\right|}, \frac{\delta}{4\left|\theta_{0}\right|}\right]$, and therefore in this case

$$
\operatorname{meas}\left\{\xi \in R\left(-\theta_{0}\right), \lambda_{1}(M(\xi)) \leq \lambda\right\}=\frac{\delta}{2\left|\theta_{0}\right|}
$$

and

$$
N\left(\theta_{0}^{2} ; H_{2}\right)=\pi^{-1} \theta_{0}
$$

for all small $\varepsilon$. The situation is the same when $|\tau|=|\nu|$ and $\left[s_{2}(0) \tau-\Re\left(\nu \bar{g}_{2}(0)\right)\right]<0$.

Now assume that $\mu=\tau \varepsilon$ with $|\tau|>|\nu|$. Then the inverse function theorem implies that (3.47) has a unique positive and a unique negative solutions $\zeta_{-}^{ \pm} \sim \pm \varepsilon$; both have a complete asymptotic expansion in powers of $\varepsilon$. In this case we have

$$
\operatorname{meas}\left\{\xi \in R\left(-\theta_{0}\right), \lambda_{1}(M(\xi)) \leq \lambda\right\}=\frac{\delta}{2\left|\theta_{0}\right|}+\sum_{p=1}^{N} \tilde{a}_{p} \varepsilon^{p}+O\left(\varepsilon^{N}\right)
$$

and

$$
N\left(\theta_{0}^{2} ; H_{2}\right)=\pi^{-1} \theta_{0}+\sum_{p=1}^{N} a_{p} \varepsilon^{p}+O\left(\varepsilon^{N}\right), \quad a_{1}<0 .
$$

Finally, let us consider the most interesting situation when $\mu=\tau \varepsilon$ with $|\tau|=|\nu|$ and $\left[s_{2}(0) \tau-\Re\left(v \bar{g}_{2}(0)\right)\right]>0$. In this case, equation (3.47) has a unique positive solution and a unique negative solution $\zeta_{-}^{ \pm} \sim \pm \varepsilon^{3 / 2}$; both $\zeta_{-}^{ \pm} \varepsilon^{-3 / 2}$ have complete asymptotic expansions in powers of $\varepsilon$. In this case we have

$$
\operatorname{meas}\left\{\xi \in R\left(-\theta_{0}\right), \lambda_{1}(M(\xi)) \leq \lambda\right\}=\frac{\delta}{2\left|\theta_{0}\right|}+\varepsilon^{3 / 2} \sum_{p=0}^{N} \tilde{a}_{p} \varepsilon^{p}+O\left(\varepsilon^{N}\right)
$$

and

$$
N\left(\theta_{0}^{2} ; H_{2}\right)=\pi^{-1} \theta_{0}+\varepsilon^{3 / 2} \sum_{p=0}^{N-2} a_{p} \varepsilon^{p}+O\left(\varepsilon^{N}\right), \quad a_{0}<0 .
$$

Case 2. Now we discuss the case $\mu<0$. In this case, as we have already mentioned, we always have

$$
\zeta^{2}+(s+\mu)-\left(4 \zeta^{2} \theta_{0}^{2}+|\nu|^{2} \varepsilon^{2}-4 t \zeta \theta_{0}+t^{2}+2 \varepsilon \Re(\nu \bar{g})+|g|^{2}\right)^{1 / 2} \leq 0
$$

whenever $|\zeta|<\frac{\delta}{4\left|\theta_{0}\right|}$ and, thus,

$$
\operatorname{meas}\left\{\xi \in R\left(-\theta_{0}\right), \lambda_{1}(M(\xi)) \leq \lambda\right\}=\frac{\delta}{2\left|\theta_{0}\right|} .
$$

Therefore, we only need to solve the inequality

$$
\zeta^{2}+(s+\mu)+\left(4 \zeta^{2} \theta_{0}^{2}+|\nu|^{2} \varepsilon^{2}-4 t \zeta \theta_{0}+t^{2}+2 \varepsilon \Re(\nu \bar{g})+|g|^{2}\right)^{1 / 2}>0 .
$$


Calculations similar to those above imply the following.

If $|\tau|<|\nu|$, or if $|\tau|=|\nu|$ and $\left[s_{2}(0) \tau-\Re\left(\nu \bar{g}_{2}(0)\right)\right]<0$, we have

$$
N\left(\theta_{0}^{2} ; H_{2}\right)=\pi^{-1} \theta_{0} \text {. }
$$

If $|\tau|>|\nu|$, we have

$$
N\left(\theta_{0}^{2} ; H_{2}\right)=\pi^{-1} \theta_{0}+\sum_{p=1}^{N} a_{p} \varepsilon^{p}+o\left(\varepsilon^{N}\right), \quad a_{1}<0 .
$$

Finally, if $|\tau|=|\nu|$ and $\varepsilon\left[s_{2}(0) \tau-\Re\left(\nu \bar{g}_{2}(0)\right)\right]>0$, we have

$$
N\left(\theta_{0}^{2} ; H_{2}\right) \sim \pi^{-1} \theta_{0}+\varepsilon^{3 / 2} \sum_{p=0}^{N-2} a_{p} \varepsilon^{p}+o\left(\varepsilon^{N}\right), \quad a_{0}<0 .
$$

The situation when $|\tau|=|\nu|$ and $\left[s_{2}(0) \tau-\Re\left(\nu \bar{g}_{2}(0)\right)\right]=0$ (in either case 1 or 2 ) can be dealt with similarly. It is not hard to see that $N\left(\theta_{0}^{2} ; H_{2}\right)$ admits the asymptotic expansion of the form

$$
N\left(\theta_{0}^{2} ; H_{2}\right) \sim \pi^{-1} \theta_{0}+\varepsilon^{k / 2} \sum_{p=0}^{N-2} a_{p} \varepsilon^{p}+o\left(\varepsilon^{N}\right), \quad a_{0}<0,
$$

with some natural $k \geq 4$ including the case $k=\infty$. We omit the details of this calculation.

Suppose now that $v=0$, so $\theta_{0} \in \Theta_{m} \backslash \Theta_{m-1}$ with $m>1$. Then we immediately have $g_{j} \equiv 0$ for all $j<m$. If $\tau \neq 0$, then we effectively have the situation discussed above, so the results will be the same (i.e. formula (3.58) holds). Assume that $\tau$ also vanishes. Then we have

$$
\sigma_{ \pm}-\theta_{0}^{2}=\zeta^{2}+s \pm\left(4 \zeta^{2} \theta_{0}^{2}-4 t \zeta \theta_{0}+t^{2}+|g|^{2}\right)^{1 / 2}=\zeta^{2}+s \pm\left(\left(2 \zeta \theta_{0}-t\right)^{2}+|g|^{2}\right)^{1 / 2} \text {. }
$$

Since $t(0)=s^{\prime}(0)=0$, it is not difficult to see that (depending on the sign of $s(0)$ ) we either always have $\sigma_{+}-\theta_{0}^{2}>0$ or $\sigma_{-}-\theta_{0}^{2}<0$. Therefore, we can repeat the above constructions. In this situation the new generic assumption will be $s_{2}(0)^{2} \neq\left|g_{2}(0)\right|^{2}$ (which for $m \geq 3$ simply means $s_{2}(0) \neq 0$ ). Then the calculations similar to those above imply that

$$
N\left(\theta_{0}^{2} ; H_{2}\right)=\pi^{-1} \theta_{0}+\sum_{p=2}^{N} a_{p} \varepsilon^{p}+o\left(\varepsilon^{N}\right), \quad a_{2}<0,
$$

for $s_{2}(0)^{2}>\left|g_{2}(0)\right|^{2}$ and (3.57) holds for $s_{2}(0)^{2}<\left|g_{2}(0)\right|^{2}$.

As above, the case $v=\tau=s_{2}(0)^{2}-\left|g_{2}(0)\right|^{2}=0$ can be considered in the same way and leads to the asymptotics (3.60) with possible value of $k \geq 5$.

The last case we have to consider is $\lambda=0$. The only points $\xi$ where there is a chance that $G(\xi)$ is negative are located in a $(1+|\tau|)^{1 / 2} \varepsilon^{1 / 2}$-neighbourhood of the origin and are not located in any resonance zone. Therefore, we have

$$
\begin{aligned}
(2 \pi) N\left(0 ; H_{2}\right) & =\operatorname{meas}\left\{\xi, \hat{h}_{2}(\xi ; 0)<0\right\} \\
& =\operatorname{meas}\left\{\xi,|\xi|^{2}+\varepsilon \tau+\sum_{p=2}^{N} \varepsilon^{p} f_{p}(\xi ; 0)+O\left(\varepsilon^{N}\right)<0\right\} .
\end{aligned}
$$


Now the simple use of the Implicit Function Theorem immediately gives the answer. If $\tau>0$, then $N\left(0 ; H_{2}\right)=0$ for small $\varepsilon$. If $\tau<0$, then

$$
N\left(0 ; H_{2}\right) \sim \varepsilon^{1 / 2}\left(\pi^{-1}|\tau|^{1 / 2}+\sum_{p=1}^{N-1} a_{p} \varepsilon^{p}\right)+O\left(\varepsilon^{N}\right) .
$$

Finally, if $\tau=0$, then we have to note that formula (3.17) implies that for small $\xi$ and non-trivial $V$ we have $f_{2}(\xi ; 0)<0$ and, therefore,

$$
N\left(0 ; H_{2}\right) \sim \varepsilon \sum_{p=0}^{N-1} a_{p} \varepsilon^{p}+O\left(\varepsilon^{N}\right), \quad a_{0}>0 .
$$

All the asymptotic formulas for $N\left(\lambda ; H_{2}\right)$ obtained above together with equations (3.2) and (3.3) lead to Theorem 2.3. Again, it is easy to see that the corresponding expansions are independent of the particular choice of the cut-off function $\varphi$.

\section{Almost-Periodic Potential}

Let us discuss the situation when the potential is not quasi-periodic, but smooth almostperiodic, i.e. $\Theta$ is still a finite set, but we have

$$
V=\sum_{\theta \in \Theta_{\infty}} \hat{V}_{\theta} \mathbf{e}_{2 \theta}
$$

with

$$
\left|\hat{V}_{\theta}\right| \ll m^{-P}
$$

for $\theta \in T_{m}$ and arbitrary positive $P$; recall that

$$
T_{m}:=\Theta_{m} \backslash \Theta_{m-1} .
$$

We also assume that $\Theta$ satisfies the diophantine condition, i.e. for $\theta \in \Theta_{m}$ we have $|\theta| \gg m^{-P_{0}}$, where $P_{0}>0$ is fixed.

Remark 4.1. We can relax the diophantine properties of the frequencies if we assume a faster decay of the Fourier coefficients: the only condition that we effectively need is that the resonance zones do not intersect, see (4.13).

The way we perform the gauge transform is, essentially, the same as in the quasiperiodic case, with one important difference: we cannot afford to have infinitely many resonance zones, therefore, before transforming the operator $H$ to $H_{1}$ and $H_{2}$ as above, we need to turn $H$ to a quasi-periodic operator by truncating the potential $V$. The level of the truncation depends on the size of $\varepsilon$ - the smaller $\varepsilon$, the more frequencies (and resonance zones) we need to keep. Thus, the number of resonance zones will be finite for each fixed $\varepsilon$, but, as opposed to the quasi-periodic situation, will increase as $\varepsilon$ goes to zero. More specifically, let us assume first that $0<\varepsilon<\varepsilon_{0}$, where $\varepsilon_{0}$ is a positive number, to be chosen later. We put $\varepsilon_{n}:=2^{-n} \varepsilon_{0}$ and $I_{n}:=\left[\frac{\varepsilon_{n}}{4}, \varepsilon_{n}\right]$. The gauge transform construction will be performed separately for each $I_{n}$ and the asymptotic expansions we will obtain will hold only for $\varepsilon \in I_{n}$. In order to 'glue' these expansions together at the end, we will use the following lemma: 
Lemma 4.2. Let $\alpha>0$ and let $F=F(\varepsilon)$ be a complex-valued function defined on $\left(0, \varepsilon_{0}\right)$. Suppose that for any natural numbers $M$ and $n$ there exists $M_{1}=M_{1}(M, n) \in \mathbb{N}$ such that for $\varepsilon \in I_{n}$ we have:

$$
F(\varepsilon)=\sum_{j=1}^{M_{1}} a_{j ; n} \varepsilon^{\alpha j}+O\left(\varepsilon_{n}^{M}\right) .
$$

Here, $a_{j ; n}$ are some coefficients depending on $j$ and $n($ and $M$ ) satisfying

$$
a_{j ; n}=O\left(\varepsilon_{n}^{-(2 \alpha j / 3)-100}\right)
$$

and the constants in the $O$-terms do not depend on $n$ (but they may depend on $M$ ). Then there exist complex numbers $\left\{a_{j}\right\}, j=1, \ldots,\left[\frac{M}{\alpha}\right]+1$ such that for all $\varepsilon, 0<\varepsilon<\varepsilon_{0}$ we have:

$$
F(\varepsilon)=\sum_{j=1}^{\left[\frac{M}{\alpha}\right]+1} a_{j} \varepsilon^{\alpha j}+O\left(\varepsilon^{M}\right) .
$$

This Lemma (in slightly different form) is proved in Section 3 of [7]; see also [6]. In order to apply it, we have to establish (4.4)-(4.5). Whenever we will be using this lemma, it will be rather straightforward to check estimates (4.5) for the coefficients from the constructions, so in what follows we will concentrate on establishing (4.4).

Remark 4.3. Note that (4.4) is not a 'proper' asymptotic formula, since the coefficients $a_{j ; n}$ are allowed to grow with $n$.

Now, we will describe the construction in more detail. Let us fix a natural number $N$ (which signifies that our errors are going to be $O\left(\varepsilon^{N}\right)$ ) and suppose that $\varepsilon \in I_{n}$. All the constructions below depend on the choice of $(n, N)$, but we will often omit writing $n$ and $N$ as the variables. Recall that for each $\theta \in \Theta_{\infty}^{\prime}$ we define $Z(\theta):=m$ for $\theta \in T_{m}$. We also fix the smoothness $P$ of the potential so that

$$
\left|\hat{V}_{\theta}\right| \ll Z(\theta)^{-P}
$$

this (large) $P$ depends on $P_{0}$ and $N$ and will be chosen later. For each natural $L$ we define the truncated potential

$$
V_{L}:=\sum_{\theta \in \Theta_{L}} \hat{V}_{\theta} \mathbf{e}_{2 \theta}
$$

Estimate (2.4) implies

$$
\left\|V-V_{L}\right\|_{\infty} \ll \sum_{Z>L} Z^{-P} \#\left(T_{Z}\right) \ll \sum_{Z>L} Z^{3 l-P} \ll L^{3 l+1-P}<L^{-P / 2},
$$

assuming of course that $P$ is sufficiently large. Now, we choose $\tilde{L}=\tilde{L}(n, N)$ so large that the norm of the operator of multiplication by $V-V_{L}$ is smaller than $\varepsilon_{n}^{N}$. The previous estimate shows that it is enough to take

$$
\tilde{L}(n ; N):=\varepsilon_{n}^{-\frac{2 N}{P}}
$$

to achieve this. Then we run $3 N$ steps of the gauge transform as described in "Appendix", but for the operator $\hat{H}_{\tilde{L}}:=H+\varepsilon V_{\tilde{L}}$. The main difference with the gauge transform 
procedure for the previous section is that now the width of each resonant zone decreases as $n$ increases. More precisely, we put

$$
R(\theta)=R_{n}(\theta):=\left\{\xi \in \mathbb{R},\left|(\xi+2 \theta)^{2}-\xi^{2}\right|<\varepsilon_{n}^{1 / 2}\right\}=\left(-\theta-\frac{\varepsilon_{n}^{1 / 2}}{4|\theta|},-\theta+\frac{\varepsilon_{n}^{1 / 2}}{4|\theta|}\right)
$$

and

$$
\varphi_{\theta}(\xi)=\varphi_{\theta ; n}(\xi):=\varphi\left((\xi+\theta) 4|\theta| \varepsilon_{n}^{-1 / 2}\right) .
$$

Then the frequencies of the resulting operator $H_{2}$ will be inside the set $\left(\Theta_{\tilde{L}}\right)_{3 N}=$ $\Theta_{3 N \tilde{L}}$.

Note that the resonant zones obtained at each step do not intersect. Indeed, suppose that $\theta_{1}, \theta_{2} \in \Theta_{3 N \tilde{L}}^{\prime}, \theta_{1} \neq \theta_{2}$. Then $\theta_{2}-\theta_{1} \in \Theta_{6 N \tilde{L}}^{\prime}$ and, therefore, our diophantine condition implies

$$
\left|\theta_{2}-\theta_{1}\right| \gg(6 N \tilde{L})^{-P_{0}} \gg \varepsilon_{n}^{\frac{3 N P_{0}}{P}}>\varepsilon_{n}^{1 / 8}
$$

for sufficiently small $\varepsilon_{n}$, assuming that $P$ is chosen so large that

$$
\frac{3 N P_{0}}{P}<1 / 8
$$

At the same time the length of the resonant zone corresponding to $\theta \in \Theta_{3 N \tilde{L}}^{\prime}$ is bounded from above by

$$
\varepsilon_{n}^{1 / 2} /|\theta| \leq \varepsilon_{n}^{1 / 2}(3 N \tilde{L})^{P_{0}} \ll \varepsilon_{n}^{1 / 2} \varepsilon_{n}^{-\frac{3 N P_{0}}{P}}<\varepsilon_{n}^{3 / 8} .
$$

Remark 4.4. Of course, condition (4.14) means that the bigger $N$ is (i.e. the more asymptotic terms we want to obtain), the bigger $P$ we should take (i.e. the smoother potentials we have to consider).

Remark 4.5. The calculation above shows that even if we consider 'wide' resonance zones which are ten times wider than (4.11) (i.e. 'wide' resonance zones are the intervals $\left.\left(-\theta-\frac{5 \varepsilon_{n}^{1 / 2}}{2|\theta|},-\theta+\frac{5 \varepsilon_{n}^{1 / 2}}{2|\theta|}\right)\right)$, then these zones will not intersect either. This observation will be useful later on.

This construction leads to two operators, $H_{1}$ and $H_{2}$ with the same properties as described in the previous section. For each $\theta \in \Theta_{3 N \tilde{L}}^{\prime}$ we denote by $R(\theta)=R(\theta ; n)$ the resonant zone-the interval centred at $-\theta$ of length $\frac{\varepsilon_{n}^{1 / 2}}{2|\theta|}$. We also denote

$$
\mathbf{R}\left(\varepsilon_{n}\right)=\mathbf{R}_{n}:=\cup_{\theta \in \Theta_{3 N \tilde{L}}^{\prime}} R(\theta ; n) ;
$$

this is the resonant zone corresponding to $I_{n}$. The meaning of this set is that the symbol $h_{2}$ of $H_{2}$ is diagonal for $\xi \notin \mathbf{R}_{n}$. This means that all Fourier coefficients $\hat{h}_{2}(\xi ; \theta)=0$ whenever $\theta \neq 0$ and $\xi \notin \mathbf{R}_{n}$; our construction implies that even more is true: $\hat{h}_{2}(\xi ; \theta)=$ 0 unless $\xi \in R(\theta ; n)$. The main difference between the almost-periodic and quasiperiodic cases is the following: in the quasi-periodic case the resonant set was fixed for any given $N$ as $\delta(N)$ and decreasing as $N$ grows (see (3.13)), whereas in the almostperiodic case $\mathbf{R}\left(\varepsilon_{n}\right)$ is fixed only when $\varepsilon \in I_{n}$, and in general it is no longer true that $\mathbf{R}_{n+1} \subset \mathbf{R}_{n}$ (since the smaller $\varepsilon_{n}$ leads to bigger $n$ and bigger $\tilde{L}(n)$ given by (4.10) and, 
thus, $\mathbf{R}_{n+1}$ consists of a bigger number of smaller zones than $\mathbf{R}_{n}$ ). Estimate (2.4) implies that the number of elements in $\Theta_{3 N \tilde{L}}$ can be estimated by

$$
(9 N \tilde{L})^{3 l} \ll \varepsilon_{n}^{-\frac{9 N l}{P}} \ll \varepsilon_{n}^{-1 / 6},
$$

which implies

$$
\operatorname{meas}\left(\mathbf{R}_{n}\right)<\varepsilon_{n}^{1 / 6}
$$

if we choose $P$ large enough.

Let us now discuss the behaviour of the gaps of $H_{2}$ (and, therefore, of $H$ ). This can be done using the arguments from the quasi-periodic case. When $\varepsilon \in I_{n}$, the operator $H_{2}$ has gaps around points $|\theta|^{2}, \theta \in \Theta_{3 N \tilde{L}(n)}$, and the length of each such gap has asymptotic expansion in natural powers of $\varepsilon$, according to Theorem 3.6. Now we notice that if $\theta \in \Theta_{3 N \tilde{L}(n)}$, then $\theta \in \Theta_{3 N \tilde{L}(m)}$ for any $m \geq n$ and, therefore, there is a gap of $H_{2}$ around $\theta$ for any $m \geq n$. The length of this gap has an asymptotic expansion given by Theorem 3.6 for $\varepsilon \in I_{m}, m \geq n$ (Here we assume that $\varepsilon_{0}$ is chosen to be small enough, depending only on $N$ ). These expansions may be different in general, but we can use Lemma 4.2 to deduce that we have a complete power asymptotic expansion of the length of a gap valid for all $\varepsilon<\varepsilon_{0}$. Thus, we obtain Theorem 2.1 for smooth almost-periodic case.

Now we discuss the asymptotic behaviour of the IDS. Recall that all our constructions are made for fixed $N$; sometimes, we will be emphasising this and make $N$ an argument of the objects we consider. First, we introduce the set of $\xi>0$ such that $\xi \notin \Theta_{\infty}$ and there is an infinite sequence $n_{j} \rightarrow \infty$ and $\theta_{j} \in \Theta_{L\left(n_{j}\right)}$ satisfying $\xi \in R\left(\theta_{j} ; n_{j}\right)$. We denote this set by $\tilde{S}_{1}(N)$. Since we have $\sum_{n=p}^{\infty} \operatorname{meas}\left(\mathbf{R}_{n}\right) \rightarrow 0$ as $p \rightarrow \infty$, the measure of $\tilde{\mathcal{S}}_{1}(N)$ is zero. Also, it is easy to see that the set $\cap_{n} \mathbf{R}_{n}(N)$ is the Cantor-type set (i.e. a perfect set with empty interior) and is, thus, uncountable (unless $V$ is periodic and $\Theta_{\infty}$ is therefore discrete). Since, obviously, $\cap_{n} \mathbf{R}_{n}(N) \subset\left(\tilde{\mathcal{S}}_{1}(N) \cup \Theta_{\infty}\right)$ and $\Theta_{\infty}$ is countable, this implies that the set $\tilde{S}_{1}(N)$ is uncountable. We also have $\tilde{S}_{1}(N) \subset \tilde{\mathcal{S}}_{1}(\tilde{N})$ for $N<\tilde{N}$. Finally, we introduce $\mathcal{S}_{1}:=\cup_{N} \tilde{S}_{1}(N)$-global uncountable set of Lebesgue measure zero.

Let us assume at the moment that $\tau=0$. For each fixed $\lambda>0$ there are the following three possibilities:

1. Let $\sqrt{\lambda} \in \Theta_{\infty}$. Then $\sqrt{\lambda}=|\theta| \in R(-|\theta| ; n)$ for all sufficiently large $n$ and we therefore can repeat the procedure from the previous section to obtain the resonance asymptotic 'expansion' (3.57) (see also Lemma 4.2).

2. Let $\sqrt{\lambda} \notin\left(\mathcal{S}_{1} \cup \Theta_{\infty}\right)$. Then for all $N$ we have $\sqrt{\lambda} \notin \tilde{S}_{1}(N) \cup \Theta_{\infty}$. Thus, for all sufficiently large $n$ we have $\lambda^{1 / 2} \notin \mathbf{R}_{n}$. Then again we can repeat the (non-resonant) procedure from the previous section which, together with Lemma 4.2, guarantees the existence of the complete asymptotic expansion (3.34).

3. Let $\sqrt{\lambda} \in \mathcal{S}_{1}$. This is the most interesting case. As we will see below, in general there is a big part of $\mathcal{S}_{1}$ where no power asymptotic expansion exists. Let us make a pause for a moment and summarize what we have done so far. We have proved the following statement:

Theorem 4.6. Suppose, $V$ is smooth almost-periodic with the constant Fourier coeffcient $\tau=0$. Then there exists a set $\mathcal{S}_{1}$ such that for $\lambda^{1 / 2} \in \mathbb{R}_{+} \backslash\left(\mathcal{S}_{1} \cup \Theta_{\infty}\right)$, we have a complete expansion of the form (3.34), whereas when $\lambda \in \Theta_{\infty}$, we have (3.57). The set $\mathcal{S}_{1}$ is uncountable and has measure zero. 
Suppose now $\tau \neq 0$. Let us denote by $R^{\prime}(\theta ; n)$ the interval centred at $-\theta$, but of twice larger length than $R(\theta ; n)$; obviously, $R(\theta ; n) \subset R^{\prime}(\theta ; n)$. We also denote by $\tilde{\mathcal{S}}_{2}(N)$ the set of points $\xi \notin \Theta_{\infty}$ for which there is an infinite sequence $n_{j} \rightarrow \infty$ and $\theta_{j} \in \Theta_{L\left(n_{j}\right)}$ such that $\xi \in R^{\prime}\left(\theta_{j} ; n_{j}\right)$. We put $\mathcal{S}_{2}=\mathcal{S}_{2}(\tau):=\cup_{N} \tilde{\mathcal{S}}_{2}(N)$. Then $\mathcal{S}_{1} \subset \mathcal{S}_{2}$, meas $\left(S_{2}\right)=0$, and for $\sqrt{\lambda} \notin \mathcal{S}_{2}$ we still have the complete asymptotic expansion. Indeed, if $\varepsilon \in I_{n_{j}}$ and $\sqrt{\lambda} \notin R^{\prime}\left(\theta_{j} ; n_{j}\right)$, then $\sqrt{\lambda+\tau \varepsilon} \notin R\left(\theta_{j} ; n_{j}\right)$ for sufficiently large $n$. This proves the following statement:

Theorem 4.7. The statements of the previous theorem hold for any $\tau \neq 0$ with the set $\mathcal{S}_{1}$ replaced by a different uncountable zero measure set $\mathcal{S}_{2}=\mathcal{S}_{2}(\tau)$.

Now we will prove the opposite-that there is a substantial set $\mathcal{S}$ such that for $\sqrt{\lambda} \in \mathcal{S}$ there is no asymptotic expansion in powers of $\varepsilon$ for $N(\lambda ; H)$. Obviously, the measure of $\mathcal{S}$ has to be zero, but we will show that it is uncountable. However, as we have seen in the previous section, such a set must be empty in the quasi-periodic case. This means that we need to make a further assumption on the potential. Namely, we will assume that $V$ is not periodic (i.e., $\Theta_{\infty}$ is dense) and $\hat{V}_{\theta} \neq 0$ for any $\theta \in \Theta_{\infty}^{\prime}$.

Remark 4.8. We can replace the last condition by requiring that there are infinitely many non-zero Fourier coefficients located in 'strategically important' places.

We again start with the case $\tau=0$. The strategy of the proof will be as follows. First, we will make a natural attempt to construct a set $\mathcal{S}$ such that for $\sqrt{\lambda} \in \mathcal{S}$ there is no asymptotic expansion in powers of $\varepsilon$ of $N(\lambda ; H)$. This attempt will almost work, but not quite. Then we will see what the problem with our first attempt is and will modify it correspondingly.

So, we define $R^{\prime \prime}(\theta ; n)=\left(-\theta-\delta_{n}(\theta),-\theta+\delta_{n}(\theta)\right)$ as the interval centred at $-\theta$ of half-length $\delta_{n}$ and at our first attempt we define $\delta_{n}(\theta)=\varepsilon_{n}\left|\hat{V}_{\theta}\right|(100|\theta|)^{-1}$; obviously, then $R^{\prime \prime}(\theta ; n) \subset R(\theta ; n)$ for large $n$. Note that our constructions guarantee that if $\xi \in R^{\prime \prime}(\theta ; n)$ and $\varepsilon \in I_{n}$, then $|\xi|^{2}$ is well inside the spectral gap of $H_{2}(n)$ (this is the operator $\mathrm{H}_{2}$, when we want to emphasize that we have performed the gauge transform for $\left.\varepsilon \in I_{n}\right)$. Now we consider the set $\tilde{S}_{3}(N)$ of all $\lambda$ for which the following two conditions are satisfied:

a. There is an infinite sequence $n_{j} \rightarrow \infty$ and $\theta_{j} \in \Theta_{3 N \tilde{L}\left(n_{j}\right)}^{\prime}$ such that $\lambda^{1 / 2} \in$ $R^{\prime \prime}\left(\theta_{j} ; n_{j}\right)$,

and

b. There is an infinite sequence $n_{j}^{\prime} \rightarrow \infty$ such that $\lambda^{1 / 2} \notin \mathbf{R}\left(n_{j}^{\prime}\right)$.

A simple argument based on the fact that $\Theta_{\infty}$ is dense in $\mathbb{R}$ implies that $\tilde{S}_{3}(N)$ is uncountable.

Suppose, $\sqrt{\lambda} \in \tilde{S}_{3}(N)$. Then, if $\varepsilon \in I_{n_{j}}$, the point $\lambda$ is in the spectral gap of $H_{2}\left(n_{j}\right)$ and, therefore, we have the (trivial) resonant asymptotic expansion (3.49). On the other hand, if $\varepsilon \in I_{n_{j}^{\prime}}$, we have the non-resonant asymptotic expansion (3.33). It is very tempting to stop the proof here by stating that these two expansions are different. However, we cannot quite guarantee this-it may well happen that all the coefficients in the non-resonant expansion (3.33) turn to zero. One way of overcoming this is to show that for generic set of Fourier coefficients of $V$ these coefficients are bounded away from zero. We, however, will assume a different strategy and reduce the set $\tilde{S}_{3}(N)$ even further (by choosing smaller values of the parameters $\delta_{n}(\theta)$ ). 
Before doing this, let us see what happens with the position of the point $\xi \in \widetilde{S}_{3}(N)$ related to different resonant zones as $n$ changes. When $n=n_{j}$, our point $\xi$ is inside the resonant zone $R^{\prime \prime}\left(\theta_{j} ; n_{j}\right)$ and, therefore, we have a trivial expansion for $\varepsilon \in I_{n_{j}}$. If we consider values $n$ bigger than $n_{j}$, then $\xi$ may stay inside $R\left(\theta_{j} ; n\right)$ for a while, but since $\cap_{n} R\left(\theta_{j} ; n\right)=\left|\theta_{j}\right| \neq \xi$, for sufficiently large $n$ our point $\xi$ will get outside of the resonant zone $R\left(\theta_{j} ; n\right)$; let us denote by $\tilde{k}_{j}$ the index when this happens (i.e. $\tilde{k}_{j}$ is smallest value of $n>n_{j}$ for which we have $\xi \notin R\left(\theta_{j} ; n\right)$ ). Similarly, let $k_{j}$ be the biggest value of $n<n_{j}$ for which we have $\xi \notin R\left(\theta_{j} ; n\right)$. Since the width of a resonance zone shrinks by a factor $\sqrt{2}$ at each step, Remark 4.5 implies that $\xi$ cannot 'enter' a different resonance zone immediately after 'leaving' $R\left(\theta_{j} ; n\right)$, i.e. $\xi \notin\left(\mathbf{R}\left(k_{j}\right) \cup \mathbf{R}\left(\tilde{k}_{j}\right)\right)$. Then by our construction we have $N$ asymptotic terms of $N\left(\lambda ; H^{(\varepsilon)}\right)$ when $\varepsilon \in I_{k_{j}}$, and the coefficient in front of $\varepsilon^{2}$ is easily computable and equal to

$$
-(2 \pi \xi)^{-1} f_{2}\left(\xi, 0 ; k_{j}\right)=(2 \pi \xi)^{-1} \sum_{\theta \in \Theta_{3 N \tilde{L}\left(k_{j}\right)}^{\prime}} \frac{\left|\hat{V}_{\theta}\right|^{2}}{|\xi+2 \theta|^{2}-|\xi|^{2}}
$$

Similarly, we have $N$ asymptotic terms of $N\left(\lambda ; H^{(\varepsilon)}\right)$ when $\varepsilon \in I_{\tilde{k}_{j}}$, and the coefficient in front of $\varepsilon^{2}$ equals

$$
-(2 \pi \xi)^{-1} f_{2}\left(\xi, 0 ; \tilde{k}_{j}\right)=(2 \pi \xi)^{-1} \sum_{\theta \in \Theta_{3 N \tilde{L}\left(\tilde{k}_{j}\right)}^{\prime}} \frac{\left|\hat{V}_{\theta}\right|^{2}}{|\xi+2 \theta|^{2}-|\xi|^{2}}
$$

Notice that the sum in (4.19) contains more terms than (4.18); one of the extra terms corresponds to $\theta=\theta_{j}$ and its modulus is at least $\frac{\varepsilon_{\tilde{k}_{j}}^{-1 / 2}\left|\hat{V}_{\theta_{j}}\right|^{2}}{9\left|\theta_{j}\right|}$. The rest of the extra terms give a total contribution of $O\left(\varepsilon_{k_{j}}^{N}\right)$. Therefore, we have

$$
\left|f_{2}\left(\xi, 0 ; \tilde{k}_{j}\right)-f_{2}\left(\xi, 0 ; k_{j}\right)\right| \geq \frac{\varepsilon_{\tilde{k}_{j}}^{-1 / 2}\left|\hat{V}_{\theta_{j}}\right|^{2}}{9\left|\theta_{j}\right|}+O\left(\varepsilon_{k_{j}}^{N}\right) .
$$

Now we will readjust the definition of the subset $R^{\prime \prime}$ of the resonant zone $R$ by requiring that the jump (4.20) is at least one, which can be achieved by asking that $\varepsilon_{\tilde{k}_{j}}^{1 / 2}<\frac{\left|\hat{V}_{\theta_{j}}\right|^{2}}{18\left|\theta_{j}\right|}$. Another way of formulation this is requesting that if $n>n_{j}$ satisfies

$$
\varepsilon_{n}^{1 / 2}>\frac{\left|\hat{V}_{\theta_{j}}\right|^{2}}{18\left|\theta_{j}\right|}
$$

then $\xi \in R\left(\theta_{j}, n\right)$. Now, we define a modified set $\tilde{S}_{3}(N)$ which satisfies properties a and $\mathrm{b}$ above, but with a modified parameter $\delta_{n}$ defining the resonant zone $R^{\prime \prime}$ given by $\delta_{n}(\theta)=\min \left\{\frac{\varepsilon_{n}\left|\hat{V}_{\theta}\right|}{100|\theta|}, \frac{\left|\hat{V}_{\theta}\right|^{2}}{72|\theta|^{2}}\right\}$. The calculations just above show that if $\xi \in R^{\prime \prime}\left(\theta_{j}, n_{j}\right)$, then, assuming once again that $\varepsilon_{0}=\varepsilon_{0}(N)$ is small enough, we have:

$$
\left|f_{2}\left(\xi, 0 ; \tilde{k}_{j}\right)-f_{2}\left(\xi, 0 ; k_{j}\right)\right| \geq 1
$$


and, therefore, we cannot have both these coefficients small at the same time. This shows that, indeed, we cannot have a complete power asymptotic expansion (nor even an asymptotic expansion with the remainder $o\left(\varepsilon^{2}\right)$ ) for any $\xi \in \tilde{S}_{3}(N)$ with $N \geq 3$.

If we put

$$
\mathcal{S}_{3}:=\cup_{N \geq 3} \tilde{\mathcal{S}}_{3}(N),
$$

then this is an uncountable set such that there is no complete power asymptotic expansion of $N(\lambda, H)$ for $\sqrt{\lambda} \in \mathcal{S}_{3}$.

We have proved the following result:

Theorem 4.9. Suppose, $V$ is smooth almost-periodic, but not periodic, the constant Fourier coefficient $\tau=0$, and $\hat{V}_{\theta} \neq 0$ for any $\theta \in \Theta_{\infty}^{\prime}$. Then there exists an uncountable set $\mathcal{S}_{3}$ such that when $\lambda^{1 / 2} \in \mathcal{S}_{3}$, there is no complete power asymptotic expansion of $N(\lambda ; H)$; even more, if $\lambda^{1 / 2} \in \mathcal{S}_{3}$, then no asymptotic expansion of $N(\lambda ; H)$ with remainder estimate o $\left(\varepsilon^{2}\right)$ exists.

Suppose now that $\tau \neq 0$. Consider the set $\tilde{S}_{3}(N)$ of all $\lambda$ for which the following two conditions are satisfied:

a. There is an infinite sequence $n_{j} \rightarrow \infty$ and $\theta_{j} \in \Theta_{3 N \tilde{L}\left(n_{j}\right)}^{\prime}$ such that $\left(\lambda+\tau \varepsilon_{n_{j}}\right)^{1 / 2} \in$ $R^{\prime \prime}\left(\theta_{j} ; n_{j}\right)$

and

b. There is an infinite sequence $n_{j}^{\prime} \rightarrow \infty$ such that $\left(\lambda+\tau \varepsilon_{n_{j}^{\prime}}\right)^{1 / 2} \notin \mathbf{R}\left(n_{j}^{\prime}\right)$.

A slightly more difficult than before (but still quite elementary) argument shows that $\tilde{S}_{3}(N)$ is uncountable for each $\tau$. Also, similar to the case $\tau=0$, if $\varepsilon \in I_{n_{j}}$, the point $\lambda$ is in the spectral gap of $H_{2}\left(n_{j}\right)$ and, therefore, we have the (trivial) resonant asymptotic expansion (3.49). On the other hand, if $\varepsilon \in I_{n_{j}^{\prime}}$, we have the non-resonant asymptotic expansion (3.33), and the coefficient at the first order term in this expression equals $-\frac{\tau}{2 \pi \sqrt{\lambda}}$, which means that these two expressions are different starting with $\varepsilon$, i.e. it is enough to take $N \geq 2$. Putting $\mathcal{S}_{3}:=\cup_{N \geq 2} \tilde{\mathcal{S}}_{3}(N)$, we will prove the analogue of Theorem 4.9 in the case $\tau \neq 0$ :

Theorem 4.10. Suppose, $V$ is smooth almost-periodic, but not periodic, the constant Fourier coefficient $\tau \neq 0$, and $\hat{V}_{\theta} \neq 0$ for any $\theta \in \Theta_{\infty}^{\prime}$. Then there exists an uncountable set $\mathcal{S}_{3}$ such that when $\lambda^{1 / 2} \in \mathcal{S}_{3}$, there is no complete power asymptotic expansion of $N(\lambda ; H)$; even more, if $\lambda^{1 / 2} \in \mathcal{S}_{3}$, then no asymptotic expansion of $N(\lambda ; H)$ with remainder estimate $o\left(\varepsilon^{1}\right)$ exists.

Putting all the results proved in this section together, we have proved the following:

Theorem 4.11. Suppose, $V$ is smooth almost-periodic, but not periodic, and $\hat{V}_{\theta} \neq 0$ for any $\theta \in \Theta_{\infty}^{\prime}$. Then there exists a set $\mathcal{S}$ (which we call a super-resonance set) such that a complete power asymptotic expansion of $N(\lambda ; H)$ exists if and only if $\lambda \notin \mathcal{S}$. The set $\mathcal{S}$ is uncountable and has measure zero.

Proof. We just notice that $\mathcal{S}_{3} \subset \mathcal{S} \subset \mathcal{S}_{1}$ for $\tau=0$ and $\mathcal{S}_{3} \subset \mathcal{S} \subset \mathcal{S}_{2}$ for $\tau \neq 0$.

Remark 4.12. We have called the set $\mathcal{S}$ the super-resonant set. An interesting question which we have not studied so far is what is the dimension of this set. 


\section{Appendix}

In this appendix, we will describe the method of gauge transform.

5.1. Preparation. Our strategy will be to find a unitary operator which reduces $H=$ $H_{0}+\varepsilon \mathrm{Op}(V), H_{0}:=-\Delta$, to another PDO, whose symbol, essentially, depends only on $\xi$ (notice that now we have started to distinguish between the potential $V$ and the operator of multiplication by it $\mathrm{Op}(V))$. More precisely, we want to find operators $H_{1}$ and $H_{2}$ with the properties discussed in Sects. 3 and 4 . The unitary operator will be constructed in the form $U=e^{i \Psi}$ with a suitable bounded self-adjoint quasi-periodic PDO $\Psi$. This is why we sometimes call it a 'gauge transform'. It is useful to consider $e^{i \Psi}$ as an element of the group

$$
U(t)=\exp \{i \Psi t\}, \quad \forall t \in \mathbb{R} .
$$

We assume that the operator $\operatorname{ad}\left(H_{0}, \Psi\right)$ is bounded, so that $U(t) D\left(H_{0}\right)=D\left(H_{0}\right)$. This assumption will be justified later on. Let us express the operator

$$
A_{t}:=U(-t) H U(t)
$$

via its (weak) derivative with respect to $t$ :

$$
A_{t}=H+\int_{0}^{t} U\left(-t^{\prime}\right) \operatorname{ad}(H ; \Psi) U\left(t^{\prime}\right) d t^{\prime} .
$$

By induction it is easy to show that

$$
\begin{aligned}
A_{1} & =H+\sum_{j=1}^{\tilde{k}} \frac{1}{j !} \operatorname{ad}^{j}(H ; \Psi)+R_{\tilde{k}+1}^{(1)}, \\
R_{\tilde{k}+1}^{(1)} & :=\int_{0}^{1} d t_{1} \int_{0}^{t_{1}} d t_{2} \ldots \int_{0}^{t_{\tilde{k}}} U\left(-t_{\tilde{k}+1}\right) \operatorname{ad}^{\tilde{k}+1}(H ; \Psi) U\left(t_{\tilde{k}+1}\right) d t_{\tilde{k}+1} .
\end{aligned}
$$

The operator $\Psi$ is sought in the form

$$
\Psi=\sum_{j=1}^{\tilde{k}} \Psi_{j}, \quad \Psi_{j}=\operatorname{Op}\left(\psi_{j}\right),
$$

with some bounded operators $\Psi_{j}$. Substitute this formula in (5.1) and rewrite, regrouping the terms:

$$
\begin{aligned}
A_{1}= & H_{0}+\varepsilon \operatorname{Op}(V)+\sum_{j=1}^{\tilde{k}} \frac{1}{j !} \sum_{l=j}^{\tilde{k}} \sum_{k_{1}+k_{2}+\cdots+k_{j}=l} \operatorname{ad}\left(H ; \Psi_{k_{1}}, \Psi_{k_{2}}, \ldots, \Psi_{k_{j}}\right) \\
& +R_{\tilde{k}+1}^{(1)}+R_{\tilde{k}+1}^{(2)}, \\
R_{\tilde{k}+1}^{(2)}:= & \sum_{j=1}^{\tilde{k}} \frac{1}{j !} \sum_{k_{1}+k_{2}+\cdots+k_{j} \geq \tilde{k}+1} \operatorname{ad}\left(H ; \Psi_{k_{1}}, \Psi_{k_{2}}, \ldots, \Psi_{k_{j}}\right) .
\end{aligned}
$$


Changing this expression yet again produces

$$
\begin{aligned}
A_{1}= & H_{0}+\varepsilon \operatorname{Op}(V)+\sum_{l=1}^{\tilde{k}} \operatorname{ad}\left(H_{0} ; \Psi_{l}\right) \\
& +\sum_{j=2}^{\tilde{k}} \frac{1}{j !} \sum_{l=j}^{\tilde{k}} \sum_{k_{1}+k_{2}+\cdots+k_{j}=l} \operatorname{ad}\left(H_{0} ; \Psi_{k_{1}}, \Psi_{k_{2}}, \ldots, \Psi_{k_{j}}\right) \\
& +\sum_{j=1}^{\tilde{k}} \frac{1}{j !} \sum_{l=j}^{\tilde{k}} \sum_{k_{1}+k_{2}+\cdots+k_{j}=l} \operatorname{ad}\left(\varepsilon \mathrm{Op}(V) ; \Psi_{k_{1}}, \Psi_{k_{2}}, \ldots, \Psi_{k_{j}}\right)+R_{\tilde{k}+1}^{(1)}+R_{\tilde{k}+1}^{(2)} .
\end{aligned}
$$

Next, we switch the summation signs and decrease $l$ by one in the second summation:

$$
\begin{aligned}
A_{1}= & H_{0}+\varepsilon \operatorname{Op}(V)+\sum_{l=1}^{\tilde{k}} \operatorname{ad}\left(H_{0} ; \Psi_{l}\right)+\sum_{l=2}^{\tilde{k}} \sum_{j=2}^{l} \frac{1}{j !} \sum_{k_{1}+k_{2}+\cdots+k_{j}=l} \operatorname{ad}\left(H_{0} ; \Psi_{k_{1}}, \Psi_{k_{2}}, \ldots, \Psi_{k_{j}}\right) \\
& +\sum_{l=2}^{\tilde{k}+1} \sum_{j=1}^{l-1} \frac{1}{j !} \sum_{k_{1}+k_{2}+\cdots+k_{j}=l-1} \operatorname{ad}\left(\varepsilon \operatorname{Op}(V) ; \Psi_{k_{1}}, \Psi_{k_{2}}, \ldots, \Psi_{k_{j}}\right)+R_{\tilde{k}+1}^{(1)}+R_{\tilde{k}+1}^{(2)} .
\end{aligned}
$$

Now we introduce the notation

$$
\begin{aligned}
B_{1} & :=\varepsilon \operatorname{Op}(V), \\
B_{l} & :=\sum_{j=1}^{l-1} \frac{1}{j !} \sum_{k_{1}+k_{2}+\cdots+k_{j}=l-1} \operatorname{ad}\left(\varepsilon \mathrm{Op}(V) ; \Psi_{k_{1}}, \Psi_{k_{2}}, \ldots, \Psi_{k_{j}}\right), \quad l \geq 2, \\
T_{l} & :=\sum_{j=2}^{l} \frac{1}{j !} \sum_{k_{1}+k_{2}+\cdots+k_{j}=l} \operatorname{ad}\left(H_{0} ; \Psi_{k_{1}}, \Psi_{k_{2}}, \ldots, \Psi_{k_{j}}\right), \quad l \geq 2 .
\end{aligned}
$$

We emphasise that the operators $B_{l}$ and $T_{l}$ depend only on $\Psi_{1}, \Psi_{2}, \ldots, \Psi_{l-1}$. Let us make one more rearrangement:

$$
\begin{aligned}
A_{1} & =H_{0}+\varepsilon \operatorname{Op}(V)+\sum_{l=1}^{\tilde{k}} \operatorname{ad}\left(H_{0}, \Psi_{l}\right)+\sum_{l=2}^{\tilde{k}} B_{l}+\sum_{l=2}^{\tilde{k}} T_{l}+R_{\tilde{k}+1}, \\
R_{\tilde{k}+1} & =B_{\tilde{k}+1}+R_{\tilde{k}+1}^{(1)}+R_{\tilde{k}+1}^{(2)} .
\end{aligned}
$$

Let $\varphi_{\theta}\left(\xi, \varepsilon_{n}\right)$ be a smooth cut-off function of the set

$$
\xi:|| \xi+\left.2 \theta\right|^{2}-|\xi|^{2} \mid>\varepsilon_{n}^{1 / 2}, \quad \varepsilon_{n}:=2^{-n} \varepsilon_{0} .
$$

More precisely, let $\psi=\psi(\xi)$ be a standard smooth non-negative cut-off function satisfying supp $\psi \subset[-1 / 2,1 / 2]$ and $\psi(\xi)=1$ for $\xi \in[-1 / 4,1 / 4]$, and let $\varphi:=1-\psi$. We put

$$
\varphi_{\theta}\left(\xi, \varepsilon_{n}\right):=\varphi\left((\xi+\theta) 4|\theta| \varepsilon_{n}^{-1 / 2}\right), \quad \varepsilon_{n}:=2^{-n} \varepsilon_{0} .
$$


For any symbol

$$
b:=\sum_{\theta} \hat{b}(\xi, \theta) \mathbf{e}_{2 \theta}(x)
$$

we use the notation

$$
b^{\natural}=b^{\natural}\left(\varepsilon_{n}\right):=\sum_{\theta} \hat{b}(\xi, \theta) \varphi_{\theta}\left(\xi, \varepsilon_{n}\right) \mathbf{e}_{2 \theta}(x) .
$$

Similar notation is used for corresponding operator, i.e. $B^{\natural}$.

Now we can specify our algorithm for finding $\Psi_{j}$ 's. The symbols $\psi_{j}$ will be found from the following system of commutator equations:

$$
\begin{aligned}
\operatorname{ad}\left(H_{0} ; \Psi_{1}\right)+B_{1}^{\natural} & =0, \\
\operatorname{ad}\left(H_{0} ; \Psi_{l}\right)+B_{l}^{\natural}+T_{l}^{\natural} & =0, l \geq 2,
\end{aligned}
$$

and hence

$$
\left\{\begin{array}{l}
A_{1}=H_{0}+Y_{\tilde{k}}-Y_{\tilde{k}}^{\natural}+R_{\tilde{k}+1}, \\
Y_{\tilde{k}}=\sum_{l=1}^{\tilde{k}} B_{l}+\sum_{l=2}^{\tilde{k}} T_{l} .
\end{array}\right.
$$

Below we denote by $y_{\tilde{k}}$ the symbol of the PDO $Y_{\tilde{k}}$. Obviously, the operators $B_{l}^{\natural}, T_{l}^{\natural}$ are bounded, and therefore, in view of (5.9), (5.10), so is the commutator $\operatorname{ad}\left(H_{0} ; \Psi\right)$. This justifies the assumption made in the beginning of the formal calculations in this section.

It is also convenient to introduce the following norm in the class of symbols:

$$
|b|:=\sum_{\theta} \sup _{\xi}|\hat{b}(\xi, \theta)| .
$$

We notice that $\|\mathrm{Op}(b)\| \leq|b|$.

\subsection{Commutator equations. Put}

$$
\tilde{\chi}_{\theta}(\xi):=\varphi_{\theta}(\xi)\left(|\xi+2 \theta|^{2}-|\xi|^{2}\right)^{-1}=\frac{\varphi_{\theta}(\xi)}{4(\xi+\theta) \theta}
$$

when $\theta \neq 0$, and $\tilde{\chi}_{0}(\xi)=0$. We have

Lemma 5.1. Let $A=\mathrm{Op}(a)$ be a symmetric PDO with symbol a such that $|a|<\infty$. Then the PDO $\Psi$ with the Fourier coefficients of the symbol $\psi(\xi, x)$ given by

$$
\hat{\psi}(\xi, \theta)=i \hat{a}(\xi, \theta) \tilde{\chi}_{\theta}(\xi)
$$

solves the equation

$$
\operatorname{ad}\left(H_{0} ; \Psi\right)+\mathrm{Op}\left(a^{\natural}\right)=0 .
$$

Moreover, the operator $\Psi$ is bounded and self-adjoint and its symbol $\psi$ satisfies the following bound:

$$
|\psi| \leq \varepsilon_{n}^{-1 / 2}|a| .
$$


Now, using Lemma 5.1, equations (5.7), (5.9), (5.10) and applying inductive arguments (cf. the proof of Lemma 4.2 from [8]), we obtain the following estimates for the symbols introduced above:

Lemma 5.2. Let $V$ be a symmetric symbol. Then $\psi_{j}, b_{j}, t_{j}$ satisfy (for $\left.\varepsilon \leq \varepsilon_{n}\right)$

$$
\begin{aligned}
\left|\psi_{j}\right| & \leq C_{j} \varepsilon_{n}^{\frac{1}{2} j}(|V|)^{j}, \quad j \geq 1 ; \\
\left|b_{j}\right|+\left|t_{j}\right| & \leq C_{j} \varepsilon_{n}^{\frac{1}{2} j+\frac{1}{2}}(|V|)^{j}, \quad j \geq 2 .
\end{aligned}
$$

Moreover, assuming $\varepsilon_{0}$ is small enough (depending on $V$ and $\tilde{k}$ ) we get

$$
\begin{aligned}
& |\psi| \ll \varepsilon_{n}^{1 / 2}|V| \\
& \left|y_{\tilde{k}}\right| \leq 2 \varepsilon_{n}|V| \\
& \left\|R_{\tilde{k}+1}\right\| \ll \varepsilon_{n}^{\frac{1}{2} \tilde{k}+1}(|V|)^{\tilde{k}+1} .
\end{aligned}
$$

Proof. The proof follows by induction. For $B_{1}$ the estimate follows from the definition and for $\Psi_{1}$ from Lemma 5.1. Now, fix $l \geq 1$ and assume (5.16) and (5.17) for all $l \geq j \geq 1$. From (5.4) we get

$$
\left|b_{l+1}\right| \leq C_{l+1} \varepsilon_{n}^{1+\frac{1}{2} l}(|V|)^{l+1}=C_{l+1} \varepsilon_{n}^{\frac{1}{2}(l+1)+\frac{1}{2}}(|V|)^{l+1} .
$$

Next, we use definition (5.4). For the first commutator we apply (5.9) or (5.10). Then assumption of the induction implies

$$
\left|t_{l+1}\right| \leq C_{l+1} \varepsilon_{n}^{\frac{1}{2} k_{1}+\frac{1}{2}+\frac{1}{2}\left(l+1-k_{1}\right)}(|V|)^{l+1}=C_{l+1} \varepsilon_{n}^{\frac{1}{2}(l+1)+\frac{1}{2}}(|V|)^{l+1} .
$$

This proves (5.17) for $j=l+1$. Applying Lemma 5.1 we get (5.16) for $j=l+1$.

Now, we take

$$
\tilde{k}>2 N
$$

Then

$$
\left\|R_{\tilde{k}+1}\right\| \ll \varepsilon_{n}^{N}
$$

and we can disregard $R_{\tilde{k}+1}$. More precisely, let $W=W_{\tilde{k}}$ be the operator with symbol

$$
w_{\tilde{k}}(\xi, x):=y_{\tilde{k}}(\xi, x)-y_{\tilde{k}}^{\natural}(\xi, x) \text {, i.e. } \hat{w}_{\tilde{k}}(\xi, \theta)=\hat{y}_{\tilde{k}}(\xi, \theta)\left(1-\varphi_{\theta}(\xi)\right) .
$$

We put $H_{1}:=A_{1}$ and $H_{2}:=-\Delta+W$. Then $\left\|H_{1}-H_{2}\right\| \ll \varepsilon_{n}^{N}$ and, moreover, the symbol $\hat{h}_{2}(\xi, \theta):=\xi^{2}+\hat{w}(\xi, \theta)$ satisfies conditions described in Sects. 3 and 4.

Remark 5.3. In the case of quasi-periodic potential $V$ the construction above can be simplified. In fact, we can use just $\delta(N)$ instead of $\varepsilon_{n}^{1 / 2}$ (see Sect. 3) and thus, avoid further "glueing" of the asymptotics for different intervals $I_{n}$ (see Lemma 4.2). Technically, it is possible because different resonant zones do not intersect for all steps up to $9 \mathrm{~N}$ and thus, the cut-off functions $\varphi_{\theta}(\xi)$ present in the symbol of the operator $H_{2}$ (see Lemma 5.4 below) are equal either to 0 or 1 for all $\xi$ close to the center of a given resonant zone or between any two neighboring resonant zones. This ensures analyticity of the functions used in the proof of (3.33) (for nonresonant $\xi$ ) and Lemma 3.5 and asymptotics below (for resonant $\xi$ ). 
5.3. Computing the symbol of the operator after gauge transform. The following lemma provides us with more explicit form of the symbol $y_{\tilde{k}}$.

Lemma 5.4. We have $\hat{y}_{\hat{k}}(\xi, \theta)=0$ for $\theta \notin \Theta_{\tilde{k}}$. Otherwise,

$$
\begin{aligned}
\hat{y}_{\tilde{k}}(\xi, \theta) & =\varepsilon \hat{V}_{\theta}+\sum_{s=1}^{\tilde{k}-1} \varepsilon^{s+1} \sum C_{s}(\xi, \theta) \hat{V}_{\theta_{s+1}} \prod_{j=1}^{s} \hat{V}_{\theta_{j}} \tilde{\chi}_{\theta_{j}^{\prime}}\left(\xi+2 \phi_{j}^{\prime}\right) \\
& =\varepsilon \hat{V}_{\theta}+\sum_{s=1}^{\tilde{k}-1} \varepsilon^{s+1} \sum C_{s}(\xi, \theta) \hat{V}_{\theta_{s+1}} \prod_{j=1}^{s} \hat{V}_{\theta_{j}} \frac{\varphi_{\theta_{j}^{\prime}}\left(\xi+2 \phi_{j}^{\prime}\right)}{4 \theta_{j}^{\prime}\left(\xi+2 \phi_{j}^{\prime}+\theta_{j}^{\prime}\right)},
\end{aligned}
$$

where the second sums are taken over all $\theta_{j} \in \Theta, \theta_{j}^{\prime}, \phi_{j}^{\prime} \in \Theta_{s+1}$ and

$$
C_{s}(\xi, \theta)=\sum_{p=1}^{s} \sum_{\theta_{j}^{\prime \prime}, \phi_{j}^{\prime \prime} \in \Theta_{s+1}} C_{s}^{(p)}(\theta) \prod_{j=j}^{p} \varphi_{\theta_{j}^{\prime \prime}}\left(\xi+2 \phi_{j}^{\prime \prime}\right) .
$$

Here $C_{s}^{(p)}(\theta)$ depend on $s, p$ and all vectors $\theta, \theta_{j}, \theta_{j}^{\prime}, \phi_{j}^{\prime}, \theta_{j}^{\prime \prime}, \phi_{j}^{\prime \prime}$. At the same time, coefficients $C_{S}^{(p)}(\theta)$ can be bounded uniformly by a constant which depends on sonly. We apply the convention that $0 / 0=0$.

The proof is identical to the proof of Lemma 9.3 from [7] and we omit it here. Explicit value of the coefficients for the second term (see (3.17) and (3.21)) can be found directly as the second order perturbation or following more carefully the first two steps of the construction for $A_{1}$ from (5.11).

Acknowledgements. We are grateful to Alexander Sobolev for reading the preliminary version of this manuscript and making useful suggestions. We are grateful to the referee for useful comments. The research of the first author was partially supported by the EPSRC Grant EP/J016829/1.

Open Access This article is distributed under the terms of the Creative Commons Attribution 4.0 International License (http://creativecommons.org/licenses/by/4.0/), which permits unrestricted use, distribution, and reproduction in any medium, provided you give appropriate credit to the original author(s) and the source, provide a link to the Creative Commons license, and indicate if changes were made.

Publisher's Note Springer Nature remains neutral with regard to jurisdictional claims in published maps and institutional affiliations.

\section{References}

1. Arnol'd, V.I.: Remarks on the perturbation theory for problems of Mathieu type. Russ. Math. Surv. 38(4), 215-233 (1983)

2. Borisov, D.I.: On the spectrum of the Schrödinger operator perturbed by a rapidly oscillating potential. J. Math. Sci. 139(1), 6243-6322 (2006)

3. Damanik, D., Goldstein, M.: On the inverse spectral problem for the quasi-periodic Schrödinger equation. Publ. Math. Inst. Hautes Etudes Sci. 119(1), 217-401 (2014)

4. Leguil, M., You, J., Zhao, Z., Zhou, Q.: Asymptotics of spectral gaps of quasi-periodic Schrödinger operators (2017). Preprint arXiv:1712.04700

5. Liu, W., Shi, Y.: Upper bounds on the spectral gaps of quasi-periodic Schrödinger operators with Liouville frequencies (2017). Preprint arXiv:1708.01760

6. Morozov, S., Parnovski, L., Shterenberg, R.: Complete asymptotic expansion of the integrated density of states of multidimensional almost-periodic pseudo-differential operators. Ann. Henri Poincaré, 15(2), 263-312 (2014) 
7. Parnovski, L., Shterenberg, R.: Complete asymptotic expansion of the integrated density of states of multidimensional almost-periodic Schrödinger operators. Ann. Math. 176(2), 1039-1096 (2012)

8. Parnovski, L., Sobolev, A.: Bethe-Sommerfeld conjecture for periodic operators with strong perturbations. Invent. Math. 181(3), 467-540 (2010)

Communicated by P. Deift 